\title{
Centralized fusion robust steady-state estimator for networked uncertain system with coloured noises, one-step random delay and packet dropouts
}

\author{
Shuang $\mathrm{Li}^{1}$, Wenqiang Liu ${ }^{1, *}$, Guili Tao ${ }^{2}$ \\ ${ }^{1}$ School of Information and Electronic Engineering (Sussex Artificial Intelligence Institute), Zhejiang Gongshang University, Hangzhou, 310018 , \\ China \\ ${ }^{2}$ College of Media Engineering, Communication University of Zhejiang, Hangzhou, 310018, China
}

\begin{abstract}
This study is aimed to address the centralized fusion (CF) robust estimation problems for a class of multisensor networked systems with mixed uncertainties, which include coloured noises, same multiplicative noises in system parameter matrices, additive white noise, uncertain noise variances, as well as the one-step random delay and packet dropouts (PD). To deal with this kind of systems, one-step random delay and inconsecutive packet dropouts are described by two Bernoulli distributed random variables. By using the augmentation, de-randomization and fictitious noise techniques, the original system has transformed into an augment model with only uncertain noise variances. In the light of the minimax robust estimation principle, based on the worst-case CF system with conservative upper bounds of uncertain noise variances, the robust CF steady-state Kalman estimators (predictor, filter, and smoother) are presented. By providing a novel approach, consist of non-negative definite matrix method and Lyapunov equation approach, prove the robustness of the CF estimator. Finally, a UPS simulation example illustrates the effectiveness of the proposed CF estimation algorithms.
\end{abstract}

Keywords: centralized fusion, multisensor networked system, coloured noises, minimax robust estimation principle , onestep random delay, packet dropouts

\section{Introduction}

\subsection{Background}

In recent years, multisensor information fusion technology has received considerable attention and has been used in many fields such as self-driving, target tracking, signal processing, and so on [1]. The problem of multisensor information fusion estimation is to utilize all local measurements from sensors, according to the optimal fusion rule to obtain the optimal fusion estimation, therefore, the performance in accuracy and stability had a significant improvement compared to the single sensor system. There are two most common information fusion techniques, the distributed fusion and the $\mathrm{CF}$, depending on whether the row date is directly used for the system [2].

Kalman filtering method is a powerful tool in estimation field since the convenience to calculate on the computer. The classical Kalman filtering method requires the fundamental condition that the model parameters and noise variances of the system are precisely known [3]. However, this condition may not always hold in many engineering applications due to some uncertainties exist in the system, such as stochastic parameters, uncertain perturbations and unmodeled dynamics. If there exist the uncertainties in the system model, the result of classical Kalman filter will not meet the expectation. One of the most wellknown approach for the uncertainties is to introduce the robust Kalman filter [4], which was selected for its reliability and validity. The key characteristic of the robust Kalman filter is that its actual filtering error variances are guaranteed to have a minimal upper bound when all of the permissible uncertainties are included.

In order to describe the stochastic parameter uncertainties, one of the most common means is multiplicative noise [5-6], which include the state-dependent and noise-dependent multiplicative noises. Various studies have traditionally relied upon a basic assumption that the state-dependent and noisedependent multiplicative noises in the system model are completely different. The current study proposes a novel condition, same multiplicative noises in system parameter matrices, which allow us to resolve the unresolved issues.

Further, it is usually assumed that the random parameter disturbance is usually white noise in the research of filtering problems of uncertain systems. However, in engineering practice, the system is often disturbed by colored noise [7]. The coloured noise is also called self-correlation noise, that is, the state of noise at each time is not independent, but correlated with the state before this time. There are generally two methods to deal with the state estimation problem with colored noise: one is to transform the system into a new form with uncorrelated noise, and then apply the estimation algorithm; the other is to directly construct a general estimation algorithm under the condition of system noise correlation [8]. At present, the research on filtering of mixed uncertain networked systems with colored noise is also one of the hot fields. In the past years, too many researchers have been studied on the coloured noises in measurement equation or state

* Corresponding author. Tel.: +86 13274502790 .

E-mail address: 1wq@zjgsu.edu.cn (W.-Q, Liu). 
equation, but few focuses on that the noises exist in both equations.

The uncertainties of noise variances can be described by deterministic uncertainties. Since the noise variance matrices are positive semidefinite, we can assume that the actual uncertain noise variances have the known conservative upper bounds.

Compared with the traditional point-to-point control mode, the networked control system reduces the system wiring, saves the system design cost, and enhances the system maintainability, interactivity and fault diagnosis ability [9]. It has been applied in many fields. Networked control has also become one of the core contents in the international control field. However, due to the limited bandwidth and energy in the communication process, it is inevitable to cause random uncertainties such as random sensor delays, packet dropouts and missing measurements. Using Bernoulli random variables with values of 0 or 1 to describe the uncertainty in networked systems is one of the common methods [10-11].

\subsection{Related work}

Up to now, many important results have been reported concerned with the robust or optimal state estimation for networked systems with multiplicative noises, colored noises, random delays, and packet dropouts [10-19]. For the stochastic uncertain systems with time-correlated fading channels and the parameter uncertainties, a robust recursive filtering be obtained via a new analysis technology [10]. However, the uncertainties of the communication channels such as package dropout and random times delay are not considered in [10]. For the stochastic uncertain networked system with additive process and measurement noises, time-correlated additive noises, and package dropout, the optimal linear recursive fullorder state estimator are proposed in [11] under the linear minimum variance (LMV) criterion by an innovation analysis approach. However, the random delay, additive noises and the noise-dependent multiplicative noises are not considered in [11]. For the time-varying discrete nonlinear dynamical networks system, a new robust state estimation algorithm is provided by taking the available information of random coupling strength and random sensor delays in [12]. However, it is limited to the time-varying nonlinear networks system. In [13], for the time-varying systems subject to non-Gaussian and timecorrelated additive measurement noises, by resorting to the Lévy-Ito theorem, a modified recursive Tobit Kalman filter be obtained. However, state-dependent and noise-dependent multiple noises are not considered in [13]. The references [10-13] are all limited to the single-sensor system, but there are also some studies on the multisensor system. For instance, for the multisensor multirate stochastic systems with correlated noises and packet dropouts, the distributed fusion filter weighted by matrices in the sense of linear unbiased minimum variance (LUMV) is addressed in [14]. However, the random delay and additive noises are not consider in [14].

Moreover, the references [10-13] focus all on the networks system, which can't reflect the reality better, and many methods in non-networked systems are also worth learning.

For the systems with one-step transmission delays, packet dropouts and time-correlated multiplicative noises, recursive state estimation algorithms are developed in the sense of minimum mean-square error in [15]. However, the noise-dependent multiplicative noises are not consideration in [15]. Additionally, in [16], the author applying a time-differencing approach to obtain recursive distributed and centralized fusion estimator for the system with multiple random uncertainties in both the sensor outputs and the transmission connections. However, the citation [16] are not consider the additive noises in the measurement equations. For the systems with multiplicative and time-correlated additive measurement noises, a convergence conditions of the optimal linear estimator be obtained in [17]. However, noise-dependent multiple noises are not considered in [17]. For the system with random parameter matrices and correlated noises, uncertain observations, sensor delays, packet dropouts, the optimal linear estimators under the least-squares optimality criterion be obtained in [18]. However, the state-dependent and noise-dependent noises are not considered in [18]. For the system with multiplicative and time-correlated additive measurement noises, an optimal estimator be obtained in [19]. However, the robustness of this estimator are not taken into account in [19].

\subsection{Innovation}

In summary, the research on robust Kalman filter for uncertain networked systems with multiplicative noise has been well studied, but the research on systems with coloured noise is rare. This study will address the robust CF filtering problem for stochastic uncertain networked systems with coloured noise, same statedependent and noise-dependent multiplicative noises, one-step random delay, packet dropouts, and uncertain noise variances. Up to now, to the best of the authors' knowledge, this research is not reported in 
existing references.

The main contributions of this paper can be highlighted as follows:

(1) The coloured noises exist in both measurement equation and state equation, which is completely different from previous system with only white noise. To deal with the coloured noises, we use an innovative method to make the coloured noises transformed into the general multiplicative noises.

(2) By using introduce vector method, the augmented method, the fictitious noise technique, as well as defining perturbation direction matrices, the original system transformed to the augmented CF system model.

(3) According to the minimax robust estimation principle, on the basis of some hypotheses, design the robust CF steady-state Kalman estimators (predictor, filter, and smoother).

(4) By employing a mixed approach, consist of non-negative definite matrix method and Lyapunov equation approach, prove the robustness of the $\mathrm{CF}$ estimator for all admissible uncertainties.

(5) A simulation example applied to the uninterruptible power system (UPS) with mixed uncertainties is given, which verifies the effectiveness and applicability of the proposed method.

\begin{tabular}{|l|l|l|l|}
\hline \multicolumn{4}{|l|}{ Nomenclature } \\
\hline$R^{n}$ & n-dimensional Euclidean space & $\operatorname{diag}(\cdot)$ & diagonal matrix \\
\hline$R^{n \times n}$ & set of $n \times n$ real matrices & $\otimes$ & Kronecker product \\
\hline $\operatorname{tr}(\Lambda)$ & trace of matrix $\Lambda$ & $\rho(\Lambda)$ & spectrum radius of matrix $\Lambda$ \\
\hline$\Lambda^{\mathrm{T}}$ & transpose of matrix $\Lambda$ & $I_{n}$ & $n$ by $n$ identity matrix \\
\hline$\Lambda^{-1}$ & inverse of matrix $\Lambda$ & $\operatorname{Prob}(\bullet)$ & occurrence probability of event “ $\bullet "$ \\
\hline $\mathrm{E}[\cdot]$ & mathematical expectation operator & 0 & zero matrix with suitable dimension \\
\hline
\end{tabular}

\section{Problem statement}

The system model to be researched are as follows:

$$
\begin{gathered}
x(t+1)=\left(\Phi+\sum_{k=1}^{q} \xi_{k}(t) \Phi_{k}\right) x(t)+\left(\Gamma+\sum_{k=1}^{q} \xi_{k}(t) \Gamma_{k}\right) w(t) \\
z_{i}(t)=\left(H_{i}+\sum_{k=1}^{q} \xi_{k}(t) H_{i k}\right) x(t)+\left(C_{i}+\sum_{k=1}^{q} \xi_{k}(t) C_{i k}\right) w(t)+g_{i}(t), i=1, \cdots, L \\
w(t+1)=D w(t)+\eta(t) \\
y_{i}(t)=\zeta_{i}(t) z_{i}(t)+\left(1-\zeta_{i}(t)\right)\left(1-\zeta_{i}(t-1)\right) z_{i}(t-1)
\end{gathered}
$$

where $t$ is the discrete time, $x(t) \in R^{n}$ is the state to be estimated, $z_{i}(t) \in R^{m_{i}}$ is the observation of $i$ th sensor, $y_{i}(t) \in R^{m_{i}}$ is the measurement received by the estimator in network, $w(t) \in R^{r}$ is the coloured noises, $g_{i}(t) \in R^{m_{i}}, i=1, \ldots, L$ are the additive white noises, $\xi_{k}(t) \in R^{1}, k=1, \ldots, q$ are the multiplicative noises. $\Phi \in R^{n \times n}, \Phi_{k} \in R^{n \times n}, \Gamma \in R^{n \times r}, \Gamma_{k} \in R^{n \times r}, H_{i} \in R^{m_{i} \times n}, H_{i k} \in R^{m_{i} \times n}, C_{i} \in R^{m_{i} \times r}, C_{i k} \in R^{m_{i} \times r}$, and $D \in R^{r \times r}$ are known constant matrices with suitable dimensions, $q$ is the number of multiplicative noises, $L$ is the number of sensors.

Hypothesis 1. $\zeta_{i}(t) \in R^{1}, i=1, \cdots, L$ are mutually uncorrelated scalar Bernoulli white noises with known probabilities $\operatorname{Prob}\left(\zeta_{i}(t)=1\right)=\varsigma_{i}, \operatorname{Prob}\left(\zeta_{i}(t)=0\right)=1-\varsigma_{i}$, where $\varsigma_{i}, i=1, \cdots, L$ are known and $0 \leq \varsigma_{i} \leq 1$, and $\zeta_{i}(t)$ are uncorrelated with other stochastic signals.

So, the following results can be got from hypothesis 1 :

$$
\mathrm{E}\left[\zeta_{i}(t)\right]=\varsigma_{i}, \mathrm{E}\left[\zeta_{i}^{2}(t)\right]=\varsigma_{i}, \mathrm{E}\left[\left(\zeta_{i}(t)-\varsigma_{i}\right)^{2}\right]=\varsigma_{i}\left(1-\varsigma_{i}\right), \mathrm{E}\left[\left(\zeta_{i}(t)-\varsigma_{i}\right)\left(\zeta_{j}(t)-\varsigma_{j}\right)\right]=0, i \neq j
$$

The aims of (4) is to describe the uncertainties in the network system, including the one-step random delay and PD. If $\zeta_{i}(t)=1$, then $y_{i}(t)=z_{i}(t)$, (no random delay and packet dropouts); if $\zeta_{i}(t)=0$ and $\zeta_{i}(t-1)=0$, then $y_{i}(t)=z_{i}(t-1) \quad$ (one-step random delay); if $\zeta_{i}(t)=0$ and $\zeta_{i}(t-1)=1$, then $y_{i}(t)=0$ (package dropout).

Hypothesis 2. $\eta(t), g_{i}(t), i=1, \cdots, L$, and $\xi_{k}(t), k=1, \cdots, q$ are mutually uncorrelated white noises with zero means and covariances as follows 


$$
\mathrm{E}\left[\left[\begin{array}{c}
\eta(t) \\
g_{i}(t) \\
\xi_{k}(t)
\end{array}\right]\left[\begin{array}{c}
\eta(u) \\
g_{j}(u) \\
\xi_{h}(u)
\end{array}\right]^{\mathrm{T}}\right]=\left[\begin{array}{ccc}
\bar{R}_{\eta} & 0 & 0 \\
0 & \bar{R}_{g_{i}} \delta_{i j} & 0 \\
0 & 0 & \bar{\sigma}_{\xi_{k}}^{2} \delta_{k h}
\end{array}\right] \delta_{t u}
$$

where $\bar{R}_{\eta}, \bar{R}_{g_{i}}$, and $\bar{\sigma}_{\xi_{k}}^{2}$ are respectively the unknown uncertain actual (true) variances of white noises $\eta(t), g_{i}(t)$, and $\xi_{k}(t) . \delta_{k j}$ denotes the Kronecker delta function, $\delta_{k k}=1, \delta_{k j}=0(k \neq j)$.

Hypothesis 3. The initial state $x(0)$ is uncorrelated with $w(t), \eta(t), g_{i}(t), \xi_{k}(t)$, and $\zeta_{i}(t)$, mathematical expectation and variance are respectively,

$$
\mathrm{E}[x(0)]=\mu_{0}, \mathrm{E}\left[\left(x(0)-\mu_{0}\right)\left(x(0)-\mu_{0}\right)^{\mathrm{T}}\right]=\bar{P}_{0}
$$

where $\mu_{0}$ is known mean of $x(0)$, and $\bar{P}_{0}$ is its unknown uncertain actual variance.

Hypothesis 4. $R_{\eta}, R_{g_{i}}, \sigma_{\xi_{k}}^{2}$, and $P_{0}$ are known conservative upper bounds of $\bar{R}_{\eta}, \bar{R}_{g_{i}}, \bar{\sigma}_{\xi_{k}}^{2}$ and $\bar{P}_{0}$, respectively, and satisfy that

$$
\bar{R}_{\eta} \leq R_{\eta}, \bar{R}_{g_{i}} \leq R_{g_{i}}, \bar{\sigma}_{\xi_{k}}^{2} \leq \sigma_{\xi_{k}}^{2}, \bar{P}_{0} \leq P_{0}
$$

Remark 1. The purpose of this paper is to design the CF robust steady-state Kalman estimators (predictor, filter, smoother) $\hat{x}_{c}(t \mid t+N)(N=-1, N=0, N>0)$ of the state $x(t)$ via "minimax robust estimate principle". If the variances in the system (1)-(4) take $\bar{R}_{\eta}=R_{\eta}, \bar{R}_{g_{i}}=R_{g_{i}}, \bar{\sigma}_{\xi_{k}}^{2}=\sigma_{\xi_{k}}^{2}$, and $\bar{P}_{0}=P_{0}$, the system (1)-(4) is called "worst-case" system. The minimax robust estimate principle means proposing the "minimum" variance estimator for the "worst-case" system.

The meaning of robustness is that the actual steady-state estimation error variances $\bar{P}_{c}(N)$ have the minimal upper bounds $P_{c}(N)$, i.e.,

$$
\bar{P}_{c}(N) \leq P_{c}(N)
$$

\section{Methods}

\subsection{Augmented CF system}

To begin this process, a new vector $\delta_{i}(t)$ should be introduced

$$
\delta_{i}(t)=\left(1-\zeta_{i}(t)\right) z_{i}(t)
$$

substituting (2) into $\delta_{i}(t)$ could be yield the detailed form as follow:

$$
\delta_{i}(t)=\left(1-\zeta_{i}(t)\right)\left(H_{i}+\sum_{k=1}^{q} \xi_{k}(t) H_{i k}\right) x(t)+\left(1-\zeta_{i}(t)\right)\left(C_{i}+\sum_{k=1}^{q} \xi_{k}(t) C_{i k}\right) w(t)+\left(1-\zeta_{i}(t)\right) g_{i}(t)
$$

also, substituting (2) into (4) and applying (8), the local measurement equation $y_{i}(t)$ convert to the following forms:

$$
y_{i}(t)=\zeta_{i}(t)\left(H_{i}+\sum_{k=1}^{q} \xi_{k}(t) H_{i k}\right) x(t)+\zeta_{i}(t)\left(C_{i}+\sum_{k=1}^{q} \xi_{k}(t) C_{i k}\right) w(t)+\zeta_{i}(t) g_{i}(t)+\left(1-\zeta_{i}(t)\right) \delta_{i}(t-1)
$$

On combing all local measurement, the $\mathrm{CF}$ measurement equation can be obtained

$$
y^{(c)}(t)=\zeta(t)\left(H^{(c)}+\sum_{k=1}^{q} \xi_{k}(t) H_{k}^{(c)}\right) x(t)+\zeta(t)\left(C^{(c)}+\sum_{k=1}^{q} \xi_{k}(t) C_{k}^{(c)}\right) w(t)+\zeta(t) g^{(c)}(t)+\left(I_{m}-\zeta(t)\right) \delta^{(c)}(t-1)
$$

where

$$
\begin{gathered}
y^{(c)}(t)=\left[y_{1}^{\mathrm{T}}(t), \cdots, y_{L}^{\mathrm{T}}(t)\right]^{\mathrm{T}}, \zeta(t)=\operatorname{diag}\left(\zeta_{1}(t) I_{m_{i}}, \cdots, \zeta_{L}(t) I_{m_{L}}\right), H^{(c)}=\left[H_{1}^{\mathrm{T}}, \cdots, H_{L}^{\mathrm{T}}\right]^{\mathrm{T}}, \\
H_{k}^{(c)}=\left[H_{1 k}^{\mathrm{T}}, \cdots, H_{L k}^{\mathrm{T}}\right]^{\mathrm{T}}, C^{(c)}=\left[C_{1}^{\mathrm{T}}, \cdots, C_{L}^{\mathrm{T}}\right]^{\mathrm{T}}, C_{k}^{(c)}=\left[C_{1 k}^{\mathrm{T}}, \cdots, C_{L k}^{\mathrm{T}}\right]^{\mathrm{T}}, \\
g^{(c)}(t)=\left[g_{1}^{\mathrm{T}}(t), \cdots, g_{L}^{\mathrm{T}}(t)\right]^{\mathrm{T}}, \delta^{(c)}(t)=\left[\delta_{1}^{\mathrm{T}}(t), \cdots, \delta_{L}^{\mathrm{T}}(t)\right]^{\mathrm{T}}, I_{m}=\operatorname{diag}\left(I_{m_{1}}, \cdots, I_{m_{L}}\right), m=\sum_{i=1}^{L} m_{i}
\end{gathered}
$$

Therefore, the augmented CF system consists of (1) and (11) as shown below

$$
\begin{gathered}
x_{a}(t+1)=\Phi_{a}(t) x_{a}(t)+\Gamma_{a}(t) w_{a}(t) \\
y^{(c)}(t)=H_{a}(t) x_{a}(t)+C_{a}(t) w_{a}(t)
\end{gathered}
$$

where 


$$
\begin{aligned}
& x_{a}(t)=\left[\begin{array}{c}
x(t) \\
w(t) \\
\delta^{(c)}(t-1)
\end{array}\right], \Phi_{a}(t)=\left[\begin{array}{ccc}
\Phi+\sum_{k=1}^{q} \xi_{k}(t) \Phi_{k} & \Gamma+\sum_{k=1}^{q} \xi_{k}(t) \Gamma_{k} & (0)_{n \times m} \\
(0)_{r \times n} & D & (0)_{r \times m} \\
\left(I_{m}-\zeta(t)\right)\left(H^{(c)}+\sum_{k=1}^{q} \xi_{k}(t) H_{k}^{(c)}\right) & \left(I_{m}-\zeta(t)\right)\left(C^{(c)}+\sum_{k=1}^{q} \xi_{k}(t) C_{k}^{(c)}\right) & (0)_{m \times m}
\end{array}\right], \\
& w_{a}(t)=\left[\begin{array}{c}
\eta(t) \\
g^{(c)}(t)
\end{array}\right], \Gamma_{a}(t)=\left[\begin{array}{cc}
(0)_{n \times r} & (0)_{n \times m} \\
I_{r} & (0)_{r \times m} \\
(0)_{m \times r} & I_{m}-\zeta(t)
\end{array}\right] \text {, } \\
& H_{a}(t)=\left[\zeta(t)\left(H^{(c)}+\sum_{k=1}^{q} \xi_{k}(t) H_{k}^{(c)}\right) \quad \zeta(t)\left(C^{(c)}+\sum_{k=1}^{q} \xi_{k}(t) C_{k}^{(c)}\right) \quad I_{m}-\zeta(t)\right], C_{a}(t)=\left[(0)_{m \times r} \quad \zeta(t)\right]
\end{aligned}
$$

Next, the mathematical expectation of $\zeta(t)$ can be calculated by utilizing (5) and (12)

$$
\Pi=\mathrm{E}[\zeta(t)]=\operatorname{diag}\left(\varsigma_{1} I_{m_{i}}, \cdots, \varsigma_{L} I_{m_{L}}\right)
$$

then, we have that for parameter matrix in augmented CF system

$$
\begin{gathered}
\Phi_{a}^{m}=\mathrm{E}\left[\Phi_{a}(t)\right]=\left[\begin{array}{ccc}
\Phi & \Gamma & (0)_{n \times m} \\
(0)_{r \times n} & D & (0)_{r \times m} \\
\left(I_{m}-\Pi\right) H^{(c)} & \left(I_{m}-\Pi\right) C^{(c)} & (0)_{m \times m}
\end{array}\right], \Gamma_{a}^{m}=\mathrm{E}\left[\Gamma_{a}(t)\right]=\left[\begin{array}{cc}
(0)_{n \times r} & (0)_{n \times m} \\
I_{r} & (0)_{r \times m} \\
(0)_{m \times r} & I_{m}-\Pi
\end{array}\right], \\
H_{a}^{m}=\mathrm{E}\left[H_{a}(t)\right]=\left[\begin{array}{lll}
\Pi H^{(c)} & \Pi C^{(c)} & I_{m}-\Pi
\end{array}\right], C_{a}^{m}=\mathrm{E}\left[C_{a}(t)\right]=\left[\begin{array}{ll}
(0)_{m \times r} & \Pi
\end{array}\right]
\end{gathered}
$$

Defining a noise as follows:

$$
\zeta_{i z}(t)=\zeta_{i}(t)-\varsigma_{i}
$$

and the statistic properties of the noises are

$$
\mathrm{E}\left[\zeta_{i z}(t)\right]=0, \sigma_{\zeta_{i z}}^{2}=\mathrm{E}\left[\zeta_{i z}(t) \zeta_{i z}^{\mathrm{T}}(t)\right]=\mathrm{E}\left[\zeta_{i z}^{2}(t)\right]=\varsigma_{i}\left(1-\varsigma_{i}\right), \mathrm{E}\left[\zeta_{i z}(t) \zeta_{j z}^{\mathrm{T}}(t)\right]=0, i \neq j
$$

obviously, $\zeta_{i z}(t)$ are white noises with zero mean.

From $(18)$ we define a new augment noise

$$
\zeta_{z}(t)=\zeta(t)-\Pi=\operatorname{diag}\left(\zeta_{1 z}(t) I_{m_{i}} \cdots, \zeta_{L z}(t) I_{m_{L}}\right)
$$

and introduce the matrices

$$
N_{i}=\operatorname{diag}\left((0)_{m_{1} \times m_{1}} \quad \cdots \quad(0)_{m_{i-1} \times m_{i-1}} I_{m_{i}}(0)_{m_{i+1} \times m_{i+1}} \cdots \quad(0)_{m_{L} \times m_{L}}\right), i=1, \cdots, L
$$

where

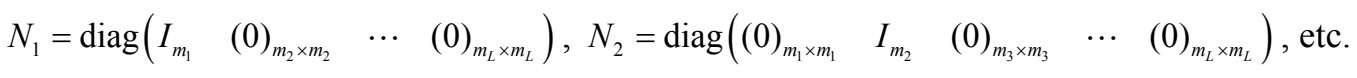

Thus, the noise $\zeta_{z}(t)$ can be rewritten as

$$
\zeta_{z}(t)=\sum_{i=1}^{L} \zeta_{i z}(t) N_{i}
$$

In order to get the random deviation items of $\Phi_{a}(t), \Gamma_{a}(t), H_{a}(t)$, and $G_{a}(t)$, we can subtract (14) from (12) after substitute $\zeta(t)=\zeta_{z}(t)+\Pi$ into (12), i.e.,

$$
\begin{gathered}
\Phi_{a}(t)-\Phi_{a}^{m}=\sum_{k=1}^{q} \xi_{k}(t) \Phi_{a}^{\xi k}+\sum_{i=1}^{L} \zeta_{i z}(t) \Phi_{a}^{\zeta i}+\sum_{i=1}^{L} \zeta_{i z}(t) \sum_{k=1}^{q} \xi_{k}(t) \Phi_{a}^{k i}, \Gamma_{a}(t)-\Gamma_{a}^{m}=\sum_{i=1}^{L} \zeta_{i z}(t) \Gamma_{a}^{\zeta i} \\
H_{a}(t)-H_{a}^{m}=\sum_{k=1}^{q} \xi_{k}(t) H_{a}^{\xi k}+\sum_{i=1}^{L} \zeta_{i z}(t) H_{a}^{\zeta i}+\sum_{i=1}^{L} \zeta_{i z}(t) \sum_{k=1}^{q} \xi_{k}(t) H_{k}^{k i}, C_{a}(t)-C_{a}^{m}=\sum_{i=1}^{L} \zeta_{i z}(t) C_{a}^{\zeta i}
\end{gathered}
$$

where

$$
\begin{aligned}
& \Phi_{a}^{\xi k}=\left[\begin{array}{ccc}
\Phi_{k} & \Gamma_{k} & (0)_{n \times m} \\
(0)_{r \times n} & (0)_{r \times r} & (0)_{r \times m} \\
\left(I_{m}-\Pi\right) H_{k}^{(c)} & \left(I_{m}-\Pi\right) C_{k}^{(c)} & (0)_{m \times m}
\end{array}\right], \Phi_{a}^{\zeta i}=\left[\begin{array}{ccc}
(0)_{n \times n} & (0)_{n \times r} & (0)_{n \times m} \\
(0)_{r \times n} & (0)_{r \times r} & (0)_{r \times m} \\
-N_{i} H^{(c)} & -N_{i} C^{(c)} & (0)_{m \times m}
\end{array}\right], \\
& \Phi_{a}^{k i}=\left[\begin{array}{ccc}
(0)_{n \times n} & (0)_{n \times r} & (0)_{n \times m} \\
(0)_{r \times n} & (0)_{r \times r} & (0)_{r \times m} \\
-N_{i} H_{k}^{(c)} & -N_{i} C_{k}^{(c)} & (0)_{m \times m}
\end{array}\right], \Gamma_{a}^{\zeta i}=\left[\begin{array}{cc}
(0)_{n \times r} & (0)_{n \times m} \\
(0)_{r \times r} & (0)_{r \times m} \\
(0)_{m \times r} & -N_{i}
\end{array}\right], H_{a}^{\xi k}=\left[\begin{array}{lll}
\Pi H_{k}^{(c)} & \Pi C_{k}^{(c)} & (0)_{m \times m}
\end{array}\right],
\end{aligned}
$$




$$
H_{a}^{\zeta i}=\left[\begin{array}{lll}
N_{i} H^{(c)} & N_{i} C^{(c)} & -N_{i}
\end{array}\right], H_{a}^{k i}=\left[\begin{array}{lll}
N_{i} H_{k}^{(c)} & N_{i} C_{k}^{(c)} & (0)_{m \times m}
\end{array}\right], C_{a}^{\zeta i}=\left[\begin{array}{ll}
(0)_{m \times r} & N_{i}
\end{array}\right]
$$

Further, the original CF system (13)-(14) has transformed into the following form which the system parameter matrixes composed by the constant parameter matrices and multiplicative noises:

$$
\begin{aligned}
& x_{a}(t+1)=\left(\Phi_{a}^{m}+\sum_{k=1}^{q} \xi_{k}(t) \Phi_{a}^{\xi k}+\sum_{i=1}^{L} \zeta_{i z}(t) \Phi_{a}^{\zeta i}+\sum_{i=1}^{L} \zeta_{i z}(t) \sum_{k=1}^{q} \xi_{k}(t) \Phi_{a}^{k i}\right) x_{a}(t)+\left(\Gamma_{a}^{m}+\sum_{i=1}^{L} \zeta_{i z}(t) \Gamma_{a}^{\zeta i}\right) w_{a}(t) \\
& y^{(c)}(t)=\left(H_{a}^{m}+\sum_{k=1}^{q} \xi_{k}(t) H_{a}^{\xi k}+\sum_{i=1}^{L} \zeta_{i z}(t) H_{a}^{\zeta i}+\sum_{i=1}^{L} \zeta_{i z}(t) \sum_{k=1}^{q} \xi_{k}(t) H_{a}^{k i}\right) x_{a}(t)+\left(C_{a}^{m}+\sum_{i=1}^{L} \zeta_{i z}(t) C_{a}^{\zeta i}\right) w_{a}(t)
\end{aligned}
$$

Lemma 1 [8]. Let $R_{i}$ be the $m_{i} \times m_{i}$ positive semi-definite matrix, i.e., $R_{i} \geq 0$, then the block-diagonal matrix $R_{\delta}=\operatorname{diag}\left(R_{1}, \cdots, R_{L}\right)$ is also positive semi-definite, i.e., $R_{\delta}=\operatorname{diag}\left(R_{1}, \cdots, R_{L}\right) \geq 0$.

The actual and conservative variances $\bar{R}_{g}^{(c)}=\operatorname{diag}\left(\bar{R}_{g_{1}}, \ldots, \bar{R}_{g_{L}}\right)$ and $R_{g}^{(c)}=\operatorname{diag}\left(R_{g_{1}}, \ldots, R_{g_{L}}\right)$ of $g^{(c)}(t)$ can be obtained from (12). Then subtracting $\bar{R}_{g}$ from $R_{g}$, and applying lemma 1 yields

$$
\bar{R}_{g}^{(c)} \leq R_{g}^{(c)}
$$

By using the (15) and (27), we can get the actual and conservative variances of white noise $w_{a}(t)$ are, respectively, given as

$$
\bar{Q}_{a}=\left[\begin{array}{cc}
\bar{R}_{\eta} & (0)_{r \times m} \\
(0)_{m \times r} & \bar{R}_{g}^{(c)}
\end{array}\right], Q_{a}=\left[\begin{array}{cc}
R_{\eta} & (0)_{r \times m} \\
(0)_{m \times r} & R_{g}^{(c)}
\end{array}\right]
$$

define $\Delta Q_{a}=Q_{a}-\bar{Q}_{a}$, and using lemma 1 , we have $\Delta Q_{a} \geq 0$, i.e.,

$$
\bar{Q}_{a} \leq Q_{a}
$$

\subsection{Actual and conservative state second non-center moments}

The actual and conservative steady-state second order non-central moments of augmented state $x_{a}(t)$ are, respectively,

$$
\begin{aligned}
\bar{X}_{a}(t+1)= & \Phi_{a}^{m} \bar{X}_{a}(t) \Phi_{a}^{m \mathrm{~T}}+\sum_{k=1}^{q} \bar{\sigma}_{\xi_{k}}^{2} \Phi_{a}^{\xi k} \bar{X}_{a}(t) \Phi_{a}^{\xi k \mathrm{~T}}+\sum_{i=1}^{L} \sigma_{\zeta_{i z}}^{2} \Phi_{a}^{\zeta i} \bar{X}_{a}(t) \Phi_{a}^{\zeta i \mathrm{~T}}+\sum_{i=1}^{L} \sigma_{\zeta_{i z}}^{2} \sum_{k=1}^{q} \bar{\sigma}_{\xi_{k}}^{2} \Phi_{a}^{k i} \bar{X}_{a}(t) \Phi_{a}^{k i \mathrm{~T}} \\
& +\Gamma_{a}^{m} \bar{Q}_{a} \Gamma_{a}^{m \mathrm{~T}}+\sum_{i=1}^{L} \sigma_{\zeta_{i z}}^{2} \Gamma_{a}^{\zeta i} \bar{Q}_{a} \Gamma_{a}^{\zeta i \mathrm{~T}} \\
X_{a}(t+1)= & \Phi_{a}^{m} X_{a}(t) \Phi_{a}^{m \mathrm{~T}}+\sum_{k=1}^{q} \sigma_{\xi_{k}}^{2} \Phi_{a}^{\xi k} X_{a}(t) \Phi_{a}^{\xi k \mathrm{~T}}+\sum_{i=1}^{L} \sigma_{\zeta_{i z}}^{2} \Phi_{a}^{\zeta i} X_{a}(t) \Phi_{a}^{\zeta \mathrm{T}}+\sum_{i=1}^{L} \sigma_{\zeta_{i z}}^{2} \sum_{k=1}^{q} \sigma_{\xi_{k}}^{2} \Phi_{a}^{k i} X_{a}(t) \Phi_{a}^{k \mathrm{~T}} \\
& +\Gamma_{a}^{m} Q_{a} \Gamma_{a}^{m \mathrm{~T}}+\sum_{i=1}^{L} \sigma_{\zeta_{i z}}^{2} \Gamma_{a}^{\zeta i} Q_{a} \Gamma_{a}^{\zeta i \mathrm{~T}}
\end{aligned}
$$

with the initial values $\quad \bar{X}_{a}(0)=\operatorname{diag}\left(\bar{X}(0) \quad(0)_{r \times r} \quad(0)_{m \times m}\right), \quad \bar{X}(0)=\bar{P}_{0}+\mu_{0} \mu_{0}^{\mathrm{T}} \quad, \quad$ and $X_{a}(0)=\operatorname{diag}\left(X(0)(0)_{r \times r}(0)_{m \times m}\right), X(0)=P_{0}+\mu_{0} \mu_{0}^{\mathrm{T}}$.

Lemma 2 [20]. $N \in R^{m \times m}$ is the positive semi-definite matrix, i.e., $N \geq 0$, then for arbitrary $Y \in R^{r \times m}$, the $r N r^{\mathrm{T}}$ is also the positive semi-definite matrix, i.e.,

$$
r N r^{\mathrm{T}} \geq 0
$$

Lemma 3. For all the unknown uncertain actual variances $\bar{Q}, \bar{R}_{\eta}$, and $\bar{P}_{0}$ satisfying (7), we have that

$$
\bar{X}_{a}(t) \leq X_{a}(t), t \geq 0
$$

Proof. It is completely similar to the proof of Lemma 5 in [21], we can prove Lemma 3, the details are omitted. The proof is completed.

Introduce the matrices $\bar{\Phi}$

$$
\bar{\Phi}=\Phi_{a}^{m} \otimes \Phi_{a}^{m}+\sum_{k=1}^{q} \bar{\sigma}_{\xi_{k}}^{2} \Phi_{a}^{\xi k} \otimes \Phi_{a}^{\xi k}+\sum_{i=1}^{L} \sigma_{\zeta_{i z}}^{2} \Phi_{a}^{\zeta i} \otimes \Phi_{a}^{\zeta i}+\sum_{i=1}^{L} \sigma_{\zeta_{i z}}^{2} \sum_{k=1}^{q} \bar{\sigma}_{\xi_{k}}^{2} \Phi_{a}^{k i} \otimes \Phi_{a}^{k i}
$$

Lemma 4. If $\Theta \in R^{m \times m}$ is the positive semi-definite matrix, i.e., $\Theta \geq 0$, and $\Theta_{\delta}=\left(\Theta_{i j}\right) \in R^{m L \times m L}$, $\Theta_{i j}=\Theta, i j=1,2, \ldots, L$, then

$$
\Theta_{\delta} \geq 0
$$

Lemma 5. For the multisensor networked system (1)-(4) with Hypotheses 1-4, if the spectrum radius 
of $\bar{\Phi}$ is less than 1 , i.e., $\rho(\bar{\Phi})<1$, then the solutions $\bar{X}_{a}(t)$ and $X_{a}(t)$ to the generalized Lyapunov equations (29) and (30) with any initial conditions $\bar{X}_{a}(0) \geq 0$ and $X_{a}(0) \geq 0$ converge respectively to the unique positive semi-definite solutions $\bar{X}_{a} \geq 0$ and $X_{a} \geq 0$ for the following generalized steady-state Lyapunov equations,

$$
\begin{aligned}
\bar{X}_{a}= & \Phi_{a}^{m} \bar{X}_{a} \Phi_{a}^{m \mathrm{~T}}+\sum_{k=1}^{q} \bar{\sigma}_{\xi_{k}}^{2} \Phi_{a}^{\xi k} \bar{X}_{a} \Phi_{a}^{\xi k \mathrm{~T}}+\sum_{i=1}^{L} \sigma_{\zeta_{i z}}^{2} \Phi_{a}^{\zeta i} \bar{X}_{a} \Phi_{a}^{\zeta i \mathrm{~T}}+\sum_{i=1}^{L} \sigma_{\zeta_{i k}}^{2} \sum_{k=1}^{q} \bar{\sigma}_{\xi_{k}}^{2} \Phi_{a}^{k i} \bar{X}_{a} \Phi_{a}^{k \mathrm{~T}} \\
& +\Gamma_{a}^{m} \bar{Q}_{a} \Gamma_{a}^{m \mathrm{~T}}+\sum_{i=1}^{L} \sigma_{\zeta_{i}}^{2} \Gamma_{a}^{\zeta i} \bar{Q}_{a} \Gamma_{a}^{\zeta i \mathrm{~T}} \\
X_{a}= & \Phi_{a}^{m} X_{a} \Phi_{a}^{m \mathrm{~T}}+\sum_{k=1}^{q} \sigma_{\xi_{k}}^{2} \Phi_{a}^{\xi k} X_{a} \Phi_{a}^{\xi k \mathrm{~T}}+\sum_{i=1}^{L} \sigma_{\zeta_{i k}}^{2} \Phi_{a}^{\zeta i} X_{a} \Phi_{a}^{\zeta i \mathrm{~T}}+\sum_{i=1}^{L} \sigma_{\zeta_{i k}}^{2} \sum_{k=1}^{q} \sigma_{\xi_{k}}^{2} \Phi_{a}^{k i} X_{a} \Phi_{a}^{k \mathrm{~T}} \\
& +\Gamma_{a}^{m} Q_{a} \Gamma_{a}^{m \mathrm{~T}}+\sum_{i=1}^{L} \sigma_{\zeta_{i}}^{2} \Gamma_{a}^{\zeta i} Q_{a} \Gamma_{a}^{\zeta i \mathrm{~T}} \\
& \lim _{t \rightarrow \infty} \bar{X}_{a}(t)=\bar{X}_{a}, \quad \lim _{t \rightarrow \infty} X_{a}(t)=X_{a}
\end{aligned}
$$

Proof. If $\rho(\bar{\Phi})<1$, similar to the proof of references [22, 23], we easily prove that (34) hold by a direct application of the result of $[24,25]$. This completes the proof.

Applying Lemma 4, as $t \rightarrow \infty$, taking the limit operations to (31) yields

$$
\bar{X}_{a} \leq X_{a}
$$

\subsection{Fictitious process and measurement noises}

A noise $w_{f}(t)$ is introduced as the fictitious process noise, which can compensate the multiplicative noise term in (25)

$$
w_{f}(t)=\left(\sum_{k=1}^{q} \xi_{k}(t) \Phi_{a}^{\xi k}+\sum_{i=1}^{L} \zeta_{i z}(t) \Phi_{a}^{\zeta i}+\sum_{i=1}^{L} \zeta_{i z}(t) \sum_{k=1}^{q} \xi_{k}(t) \Phi_{a}^{k i}\right) x_{a}(t)+\left(\Gamma_{a}^{m}+\sum_{i=1}^{L} \zeta_{i z}(t) \Gamma_{a}^{\zeta i}\right) w_{a}(t)
$$

thus, (25) can be rewritten as

$$
x_{a}(t+1)=\Phi_{a}^{m} x_{a}(t)+w_{f}(t)
$$

$w_{f}(t)$ is white noise with zero mean, and actual and conservative steady-state variances are, respectively,

$$
\begin{aligned}
& \bar{Q}_{f}=\sum_{k=1}^{q} \bar{\sigma}_{\xi_{k}}^{2} \Phi_{a}^{\xi k} \bar{X}_{a} \Phi_{a}^{\xi k \mathrm{~T}}+\sum_{i=1}^{L} \sigma_{\zeta_{i z}}^{2} \Phi_{a}^{\zeta i} \bar{X}_{a} \Phi_{a}^{\zeta i \mathrm{~T}}+\sum_{i=1}^{L} \sigma_{\zeta_{i}}^{2} \sum_{k=1}^{q} \bar{\sigma}_{\xi_{k}}^{2} \Phi_{a}^{k i} \bar{X}_{a} \Phi_{a}^{k \mathrm{~T} \mathrm{~T}}+\Gamma_{a}^{m} \bar{Q}_{a} \Gamma_{a}^{m \mathrm{~T}}+\sum_{i=1}^{L} \sigma_{\zeta_{i z}}^{2} \Gamma_{a}^{\zeta i} \bar{Q}_{a} \Gamma_{a}^{\zeta \mathrm{T}} \\
& Q_{f}=\sum_{k=1}^{q} \sigma_{\xi_{k}}^{2} \Phi_{a}^{\xi k} X_{a} \Phi_{a}^{\xi k \mathrm{~T}}+\sum_{i=1}^{L} \sigma_{\zeta_{i z}}^{2} \Phi_{a}^{\zeta i} X_{a} \Phi_{a}^{\zeta i \mathrm{~T}}+\sum_{i=1}^{L} \sigma_{\zeta_{i z}}^{2} \sum_{k=1}^{q} \sigma_{\xi_{k}}^{2} \Phi_{a}^{k i} X_{a} \Phi_{a}^{k \mathrm{~T}}+\Gamma_{a}^{m} Q_{a} \Gamma_{a}^{m \mathrm{~T}}+\sum_{i=1}^{L} \sigma_{\zeta_{i z}}^{2} \Gamma_{a}^{\zeta i} Q_{a} \Gamma_{a}^{\zeta i \mathrm{~T}}
\end{aligned}
$$

Substituting $\sigma_{\xi_{k}}^{2}=\bar{\sigma}_{\xi_{k}}^{2}+\Delta \sigma_{\xi_{k}}^{2}$ into (39), then subtracting (38) from (39) yields

$$
\begin{aligned}
\Delta Q_{f}= & \sum_{k=1}^{q} \bar{\sigma}_{\xi_{k}}^{2} \Phi_{a}^{\xi k} \Delta X_{a} \Phi_{a}^{\xi k \mathrm{~T}}+\sum_{k=1}^{q} \Delta \sigma_{\xi_{k}}^{2} \Phi_{a}^{\xi k} X_{a} \Phi_{a}^{\xi k \mathrm{~T}}+\sum_{i=1}^{L} \sigma_{\zeta_{i z}}^{2} \Phi_{a}^{\zeta i} \Delta X_{a} \Phi_{a}^{\zeta \mathrm{T} \mathrm{T}}+\sum_{i=1}^{L} \sigma_{\zeta_{i k}}^{2} \sum_{k=1}^{q} \bar{\sigma}_{\xi_{k}}^{2} \Phi_{a}^{k i} \Delta X_{a} \Phi_{a}^{k \mathrm{~T}} \\
& +\sum_{i=1}^{L} \sigma_{\zeta_{i z}}^{2} \sum_{k=1}^{q} \Delta \sigma_{\xi_{k}}^{2} \Phi_{a}^{k i} X_{a} \Phi_{a}^{k \mathrm{~T} \mathrm{~T}}+\Gamma_{a}^{m} \Delta Q_{a} \Gamma_{a}^{m \mathrm{~T}}+\sum_{i=1}^{L} \sigma_{\zeta_{i z}}^{2} \Gamma_{a}^{\zeta i} \Delta Q_{a} \Gamma_{a}^{\zeta i \mathrm{~T}}
\end{aligned}
$$

applying (28) and (35), it is easy to prove that $\Delta Q_{f} \geq 0$, i.e.,

$$
\bar{Q}_{f} \leq Q_{f}
$$

Similarly, the $v_{f}$ is introduced as the fictitious measurement noise and compensates the multiplicative noise term in (26)

$$
v_{f}=\left(\sum_{k=1}^{q} \xi_{k}(t) H_{a}^{\xi k}+\sum_{i=1}^{L} \zeta_{i z}(t) H_{a}^{\zeta i}+\sum_{i=1}^{L} \zeta_{i z}(t) \sum_{k=1}^{q} \xi_{k}(t) H_{a}^{k i}\right) x_{a}(t)+\left(C_{a}^{m}+\sum_{i=1}^{L} \zeta_{i z}(t) C_{a}^{\zeta i}\right) w_{a}(t)
$$

then (26) becomes

$$
y^{(c)}(t)=H_{a}^{m} x_{a}(t)+v_{f}(t)
$$

$v_{f}$ is white noise with zero mean, and the actual and conservative steady-state variances are, respectively,

$$
\bar{R}_{f}=\sum_{k=1}^{q} \bar{\sigma}_{\xi_{k}}^{2} H_{a}^{\xi k} \bar{X}_{a} H_{a}^{\xi k \mathrm{~T}}+\sum_{i=1}^{L} \sigma_{\zeta_{i z}}^{2} H_{a}^{\zeta i} \bar{X}_{a} H_{a}^{\zeta i \mathrm{~T}}+\sum_{i=1}^{L} \sigma_{\zeta_{i}}^{2} \sum_{k=1}^{q} \bar{\sigma}_{\xi_{k}}^{2} H_{a}^{k i} \bar{X}_{a} H_{a}^{k \mathrm{~T}}+C_{a}^{m} \bar{Q}_{a} C_{a}^{m \mathrm{~T}}+\sum_{i=1}^{L} \sigma_{\zeta_{i z}}^{2} C_{a}^{\zeta i} \bar{Q}_{a} C_{a}^{\zeta \mathrm{T}}
$$




$$
R_{f}=\sum_{k=1}^{q} \sigma_{\xi_{k}}^{2} H_{a}^{\xi k} X_{a} H_{a}^{\xi k \mathrm{~T}}+\sum_{i=1}^{L} \sigma_{\zeta_{i z}}^{2} H_{a}^{\zeta i} X_{a} H_{a}^{\zeta \mathrm{T} T}+\sum_{i=1}^{L} \sigma_{\zeta_{i z}}^{2} \sum_{k=1}^{q} \sigma_{\xi_{k}}^{2} H_{a}^{k i} X_{a} H_{a}^{k i \mathrm{~T}}+C_{a}^{m} Q_{a} C_{a}^{m \mathrm{~T}}+\sum_{i=1}^{L} \sigma_{\zeta_{i z}}^{2} C_{a}^{\zeta i} Q_{a} C_{a}^{\zeta \mathrm{T}}
$$

Similar to the computing processing of (40), subtracting (44) from (45) yields

$$
\begin{aligned}
\Delta R_{f}= & \sum_{k=1}^{q} \bar{\sigma}_{\xi_{k}}^{2} H_{a}^{\xi k} \Delta X_{a} H_{a}^{\xi k \mathrm{~T}}+\sum_{k=1}^{q} \Delta \sigma_{\xi_{k}}^{2} H_{a}^{\xi k} X_{a} H_{a}^{\xi k \mathrm{~T}}+\sum_{i=1}^{L} \sigma_{\zeta_{i z}}^{2} H_{a}^{\zeta i} \Delta X_{a} H_{a}^{\zeta i \mathrm{~T}}+\sum_{i=1}^{L} \sigma_{\zeta_{i z}}^{2} \sum_{k=1}^{q} \bar{\sigma}_{\xi_{k}}^{2} H_{a}^{k i} \Delta X_{a} H_{a}^{k i \mathrm{~T}} \\
& +\sum_{i=1}^{L} \sigma_{\zeta_{i z}}^{2} \sum_{k=1}^{q} \Delta \sigma_{\xi_{k}}^{2} H_{k}^{k i} X_{a} H_{k}^{k i \mathrm{~T}}+C_{a}^{m} \Delta Q_{a} C_{a}^{m \mathrm{~T}}+\sum_{i=1}^{L} \sigma_{\zeta_{i z}}^{2} C_{a}^{\zeta i} \Delta Q_{a} C_{a}^{\zeta \mathrm{T}}
\end{aligned}
$$

and it is easy to prove that

$$
\bar{R}_{f} \leq R_{f}
$$

The actual and conservative steady-state correlate matrices of $w_{f}(t)$ and $v_{f}(t)$ are respectively, computed by

$$
\begin{aligned}
& \bar{S}_{f}=\sum_{k=1}^{q} \bar{\sigma}_{\xi_{k}}^{2} \Phi_{a}^{\xi k} \bar{X}_{a} H_{a}^{\xi k \mathrm{~T}}+\sum_{i=1}^{L} \sigma_{\zeta_{i z}}^{2} \Phi_{a}^{\zeta i} \bar{X}_{a} H_{a}^{\zeta i \mathrm{~T}}+\sum_{i=1}^{L} \sigma_{\zeta_{i z}}^{2} \sum_{k=1}^{q} \bar{\sigma}_{\xi_{k}}^{2} \Phi_{a}^{k i} \bar{X}_{a} H_{a}^{k i \mathrm{~T}}+\Gamma_{a}^{m} \bar{Q}_{a} C_{a}^{m \mathrm{~T}}+\sum_{i=1}^{L} \sigma_{\zeta_{i z}}^{2} \Gamma_{a}^{\zeta i} \bar{Q}_{a} C_{a}^{\zeta i \mathrm{~T}} \\
& S_{f}=\sum_{k=1}^{q} \sigma_{\xi_{k}}^{2} \Phi_{a}^{\xi k} X_{a} H_{a}^{\xi k \mathrm{~T}}+\sum_{i=1}^{L} \sigma_{\zeta_{i z}}^{2} \Phi_{a}^{\zeta i} X_{a} H_{a}^{\zeta i \mathrm{~T}}+\sum_{i=1}^{L} \sigma_{\zeta_{i z}}^{2} \sum_{k=1}^{q} \sigma_{\xi_{k}}^{2} \Phi_{a}^{k i} X_{a} H_{a}^{k i \mathrm{~T}}+\Gamma_{a}^{m} Q_{a} C_{a}^{m \mathrm{~T}}+\sum_{i=1}^{L} \sigma_{\zeta_{i z}}^{2} \Gamma_{a}^{\zeta i} Q_{a} C_{a}^{\zeta \mathrm{T}}
\end{aligned}
$$

then subtracting (44) from (45) yields

$$
\begin{aligned}
\Delta S_{f} & =\sum_{k=1}^{q} \bar{\sigma}_{\xi_{k}}^{2} \Phi_{a}^{\xi k} \Delta X_{a} H_{a}^{\xi k \mathrm{~T}}+\sum_{k=1}^{q} \Delta \sigma_{\xi_{k}}^{2} \Phi_{a}^{\xi k} X_{a} H_{a}^{\xi k \mathrm{~T}}+\sum_{i=1}^{L} \sigma_{\zeta_{i}}^{2} \Phi_{a}^{\zeta i} \Delta X_{a} H_{a}^{\zeta \mathrm{T}}+\sum_{i=1}^{L} \sigma_{\zeta_{i k}}^{2} \sum_{k=1}^{q} \bar{\sigma}_{\xi_{k}}^{2} \Phi_{a}^{k i} \Delta X_{a} H_{a}^{k i \mathrm{~T}} \\
& +\sum_{i=1}^{L} \sigma_{\zeta_{i z}}^{2} \sum_{k=1}^{q} \Delta \sigma_{\xi_{k}}^{2} \Phi_{a}^{k i} X_{a} H_{a}^{k i \mathrm{~T}}+\Gamma_{a}^{m} \Delta Q_{a} C_{a}^{m \mathrm{~T}}+\sum_{i=1}^{L} \sigma_{\zeta_{i z}}^{2} \Gamma_{a}^{\zeta i} \Delta Q_{a} C_{a}^{\zeta \mathrm{T} \mathrm{T}}
\end{aligned}
$$

Hypothesis 5. Assume that the pair $\left(\Phi_{a}^{m}, H_{a}^{m}\right)$ is completely detectable, and the pair $(\bar{\Phi}, \Upsilon)$ is completely stabilizable, where $\bar{\Phi}=\Phi_{a}^{m}-S H_{a}^{m}, S=S_{f} R_{f}^{-1}, r \gamma^{\mathrm{T}}=Q_{f}-S_{f} R_{f}^{-1} S_{f}^{\mathrm{T}}$.

\section{Results}

\subsection{Robust CF steady-state Kalman predictors}

Under the conditions of hypotheses 1-5, for the worst-case time-invariant CF system (37) and (43) with known conservative noise statistics $Q_{f}, R_{f}$, and $S_{f}$, based on the minimax robust estimation principle, applying the standard Kalman filtering algorithm [6] yields the steady-state one-step Kalman predictors as

$$
\begin{gathered}
\hat{x}_{a}(t+1 \mid t)=\Psi_{a p} \hat{x}_{a}(t \mid t-1)+K_{a p} y^{(c)}(t) \\
\varepsilon_{a}(t)=y^{(c)}(t)-H_{a}^{m} \hat{x}_{a}(t \mid t-1) \\
\Psi_{a p}=\Phi_{a}^{m}-K_{a p} H_{a}^{m}, K_{a p}=\left[\Phi_{a}^{m} P_{a}(-1) H_{a}^{m \mathrm{~T}}+S_{f}\right] Q_{\varepsilon a}^{-1}, Q_{\varepsilon a}=H_{a}^{m} P_{a}(-1) H_{a}^{m \mathrm{~T}}+R_{f}
\end{gathered}
$$

with the initial value $\hat{x}_{a}(0 \mid-1)=\left[\begin{array}{lll}\mu_{0}^{\mathrm{T}} & \left((0)_{r \times 1}\right)^{\mathrm{T}} & \left((0)_{m \times 1}\right)^{\mathrm{T}}\end{array}\right]^{\mathrm{T}}$.

The conservative steady-state prediction error variances $P_{a}(-1)$ satisfies the following steady-state Riccati equation

$$
P_{a}(-1)=\Phi_{a}^{m} P_{a}(-1) \Phi_{a}^{m \mathrm{~T}}-\left[\Phi_{a}^{m} P_{a}(-1) H_{a}^{m \mathrm{~T}}+S_{f}\right]\left[H_{a}^{m} P_{a}(-1) H_{a}^{m \mathrm{~T}}+R_{f}\right]^{-1}\left[\Phi_{a}^{m} P_{a}(-1) H_{a}^{m \mathrm{~T}}+S_{f}\right]^{\mathrm{T}}+Q_{f}
$$

Remark 2. The local measurement $y_{i}(t)$, produced by the "worst-case" system (1)-(4), are called the conservative local measurements, and are unavailable (unknown). Thus, the conservative $\mathrm{CF}$ measurements $y^{(c)}(t)$, consisted by $y_{i}(t)$, are also unavailable. While the measurements $y_{i}(t)$ generated from the actual system (1)-(4) with the actual variances $\bar{R}_{\eta}, \bar{R}_{g_{i}}, \bar{\sigma}_{\xi_{k}}^{2}$, and $\bar{P}_{0}$ are called the actual measurements, and are available (known). Further, the actual CF measurements $y^{(c)}(t)$ given by (12) with the actual local measurements $y_{i}(t)$ are available. When we adopt the conservative CF measurements $y^{(c)}(t)$ to the actual CF measurements $y^{(c)}(t)$, the actual CF Kalman predictor can be obtained.

The steady-state prediction error is $\tilde{x}_{a}(t+1 \mid t)=x_{a}(t+1)-\hat{x}_{a}(t+1 \mid t)$, then subtracting (51) from (37) yields 


$$
\tilde{x}_{a}(t+1 \mid t)=\Psi_{a p} \tilde{x}_{a}(t \mid t-1)+w_{f}(t)-K_{a p} v_{f}(t)=\Psi_{a p} \tilde{x}_{a}(t \mid t-1)+\left[I_{n+m},-K_{a p}\right] \lambda_{f}(t)
$$

Define the augmented noises $\lambda_{f}(t)$ as

$$
\lambda_{f}(t)=\left[\begin{array}{ll}
w_{f}^{\mathrm{T}}(t) & v_{f}^{\mathrm{T}}(t)
\end{array}\right]^{\mathrm{T}}
$$

the actual and conservative steady-state covariances of augmented noises $\lambda_{f}(t)$ are, respectively,

$$
\bar{\Lambda}=\left[\begin{array}{ll}
\bar{Q}_{f} & \bar{S}_{f} \\
\bar{S}_{f}^{\mathrm{T}} & \bar{R}_{f}
\end{array}\right], \Lambda=\left[\begin{array}{ll}
Q_{f} & S_{f} \\
S_{f}^{\mathrm{T}} & R_{f}
\end{array}\right]
$$

Further, the actual and conservative CF steady-state prediction error covariances satisfy the following Lyapunov equations, respectively,

$$
\begin{aligned}
& \bar{P}_{a}(-1)=\Psi_{a p} \bar{P}_{a}(-1) \Psi_{a p}^{\mathrm{T}}+\left[I_{n+r+m},-K_{a p}\right] \bar{\Lambda}_{f}\left[I_{n+r+m},-K_{a p}\right]^{\mathrm{T}} \\
& P_{a}(-1)=\Psi_{a p} P_{a}(-1) \Psi_{a p}^{\mathrm{T}}+\left[I_{n+r+m},-K_{a p}\right] \Lambda_{f}\left[I_{n+r+m},-K_{a p}\right]^{\mathrm{T}}
\end{aligned}
$$

Lemma 6. For all the admissible uncertain actual variances $\bar{R}_{\eta}, \bar{R}_{g_{i}}, \bar{\sigma}_{\xi_{k}}^{2}$, and $\bar{P}_{0}$, we have that

$$
\bar{\Lambda}_{f} \leq \Lambda_{f}
$$

Proof. Define $\Delta \Lambda_{f}=\Lambda_{f}-\bar{\Lambda}_{f}, \Delta Q_{a}=Q_{a}-\bar{Q}_{a}$, and $\Delta X_{a}=X_{a}-\bar{X}_{a}$, then using (40), (46), and (50), we have that

$$
\begin{aligned}
& \Delta \Lambda_{f}=\left[\begin{array}{ll}
\Delta Q_{f} & \Delta S_{f} \\
\Delta S_{f}^{\mathrm{T}} & \Delta R_{f}
\end{array}\right]
\end{aligned}
$$

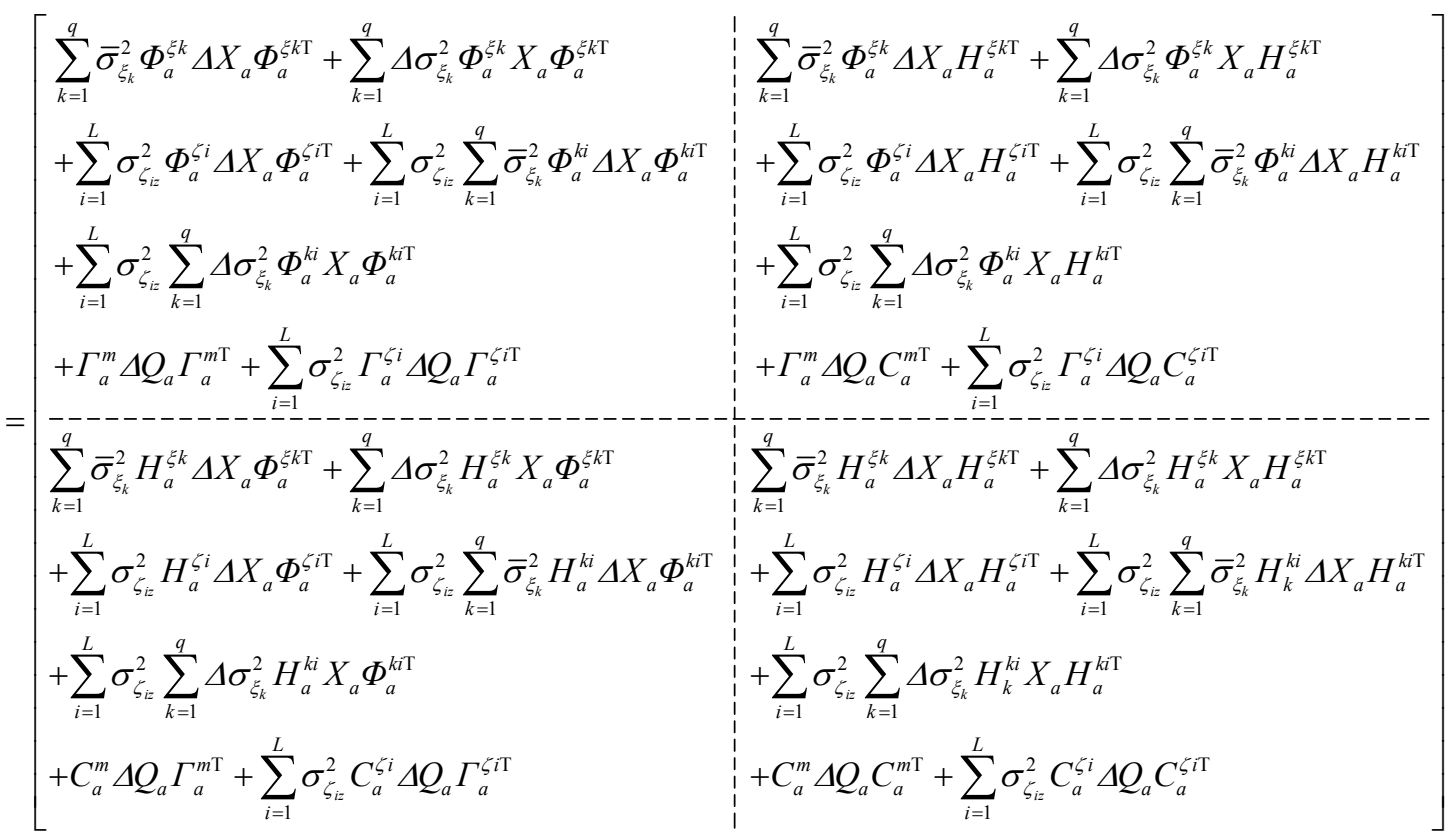

Partition $\Delta \Lambda_{f}$ into $\Delta \Lambda_{f}=\Delta \Lambda_{f}^{(1)}+\Delta \Lambda_{f}^{(2)}+\ldots+\Delta \Lambda_{f}^{(7)}$

with the definitions:

$$
\begin{gathered}
\Delta \Lambda_{f}^{(1)}=\left[\begin{array}{c:c:c}
\sum_{k=1}^{q} \bar{\sigma}_{\xi_{k}}^{2} \Phi_{a}^{\xi k} \Delta X_{a} \Phi_{a}^{\xi k \mathrm{~T}} & \sum_{k=1}^{q} \bar{\sigma}_{\xi_{k}}^{2} \Phi_{a}^{\xi k} \Delta X_{a} H_{a}^{\xi k \mathrm{~T}} \\
\hdashline \sum_{k=1}^{q} \bar{\sigma}_{\xi_{k}}^{2} H_{a}^{\xi k} \Delta X_{a} \Phi_{a}^{\xi k \mathrm{~T}} & \sum_{k=1}^{q} \bar{\sigma}_{\xi_{k}}^{2} H_{a}^{\xi k} \Delta X_{a} H_{a}^{\xi k \mathrm{~T}}
\end{array}\right], \Delta \Lambda_{f}^{(2)}=\left[\begin{array}{c:c}
\sum_{k=1}^{q} \Delta \sigma_{\xi_{k}}^{2} \Phi_{a}^{\xi k} X_{a} \Phi_{a}^{\xi k \mathrm{~T}} & \sum_{k=1}^{q} \Delta \sigma_{\xi_{k}}^{2} \Phi_{a}^{\xi k} X_{a} H_{a}^{\xi k \mathrm{~T}} \\
\hdashline \sum_{k=1}^{q} \Delta \sigma_{\xi_{k}}^{2} H_{a}^{\xi k} X_{a} \Phi_{a}^{\xi k \mathrm{~T}} & \sum_{k=1}^{q} \Delta \sigma_{\xi_{k}}^{2} H_{a}^{\xi k} X_{a} H_{a}^{\xi k \mathrm{~T}}
\end{array}\right], \\
\Delta \Lambda_{f}^{(3)}=\left[\begin{array}{c:c}
\sum_{i=1}^{L} \sigma_{\zeta_{i z}}^{2} \Phi_{a}^{\zeta i} \Delta X_{a} \Phi_{a}^{\zeta i \mathrm{~T}} & \sum_{i=1}^{L} \sigma_{\zeta_{i}}^{2} \Phi_{a}^{\zeta i} \Delta X_{a} H_{a}^{\zeta i \mathrm{~T}} \\
\hdashline \sum_{i=1}^{L} \sigma_{\zeta_{i}}^{2} H_{a}^{\zeta i} \Delta X_{a} \Phi_{a}^{\zeta \mathrm{T} \mathrm{T}} & \sum_{i=1}^{L} \sigma_{\zeta_{i z}}^{2} H_{a}^{\zeta i} \Delta X_{a} H_{a}^{\zeta i \mathrm{~T}}
\end{array}\right],
\end{gathered}
$$




$$
\begin{aligned}
& \Delta \Lambda_{f}^{(4)}=\left[\begin{array}{c:c}
\sum_{i=1}^{L} \sigma_{\zeta_{i z}}^{2} \sum_{k=1}^{q} \bar{\sigma}_{\xi_{k}}^{2} \Phi_{a}^{k i} \Delta X_{a} \Phi_{a}^{k \mathrm{~T}} & \sum_{i=1}^{L} \sigma_{\zeta_{i k}}^{2} \sum_{k=1}^{q} \bar{\sigma}_{\xi_{k}}^{2} \Phi_{a}^{k i} \Delta X_{a} H_{a}^{k \mathrm{~T}} \\
\hdashline \sum_{i=1}^{L} \sigma_{\zeta_{i k}}^{2} \sum_{k=1}^{q} \bar{\sigma}_{\xi_{k}}^{2} H_{a}^{k i} \Delta X_{a} \Phi_{a}^{k i \mathrm{~T}} & \sum_{i=1}^{L} \sigma_{\zeta_{i z}}^{2} \sum_{k=1}^{q} \bar{\sigma}_{\xi_{k}}^{2} H_{a}^{k i} \Delta X_{a} H_{a}^{k i \mathrm{~T}}
\end{array}\right],
\end{aligned}
$$

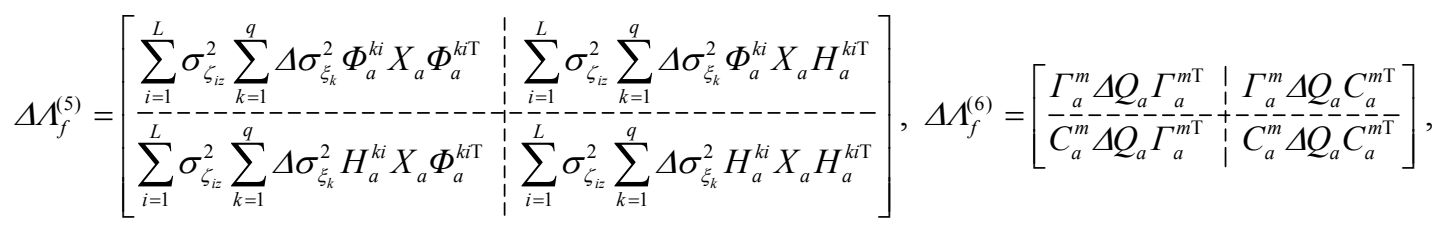

$$
\begin{aligned}
& \Delta \Lambda_{f}^{(7)}=\left[\begin{array}{c:c}
\sum_{i=1}^{L} \sigma_{\zeta_{i z}}^{2} \Gamma_{a}^{\zeta i} \Delta Q_{a} \Gamma_{a}^{\zeta \mathrm{T}} & \sum_{i=1}^{L} \sigma_{\zeta_{i z}}^{2} \Gamma_{a}^{\zeta i} \Delta Q_{a} C_{a}^{\zeta \mathrm{T}} \\
\hdashline \sum_{i=1}^{L} \sigma_{\zeta_{i z}}^{2} C_{a}^{\zeta i} \Delta Q_{a} \Gamma_{a}^{\zeta \mathrm{T}} & \sum_{i=1}^{L} \sigma_{\zeta_{i z}}^{2} C_{a}^{\zeta i} \Delta Q_{a} C_{a}^{\zeta i \mathrm{~T}}
\end{array}\right]
\end{aligned}
$$

We are already known that from (35) $\Delta X_{a} \geq 0$, and $X_{a} \geq 0$ according to the semi-positive characterization of variance matrix. Then using Lemma 4 , we can get that

$$
\Delta X_{f}=\left[\begin{array}{cc}
\Delta X_{a} & \Delta X_{a} \\
\Delta X_{a} & \Delta X_{a}
\end{array}\right] \geq 0, X_{f}=\left[\begin{array}{cc}
X_{a} & X_{a} \\
X_{a} & X_{a}
\end{array}\right] \geq 0
$$

Further, $\Delta \Lambda_{f}^{(1)}, \Delta \Lambda_{f}^{(2)}, \Delta \Lambda_{f}^{(3)}, \Delta \Lambda_{f}^{(4)}$, and $\Delta \Lambda_{f}^{(5)}$ can be rewritten as, respectively,

where

$$
\begin{gathered}
\Delta \Lambda_{f}^{(1)}=\sum_{k=1}^{q} \bar{\sigma}_{\xi_{k}}^{2} B_{a}^{\xi k} \Delta X_{f} B_{a}^{\xi k \mathrm{~T}}, \Delta \Lambda_{f}^{(2)}=\sum_{k=1}^{q} \Delta \sigma_{\xi_{k}}^{2} B_{a}^{\xi k} X_{a} B_{a}^{\xi k \mathrm{~T}}, \Delta \Lambda_{f}^{(3)}=\sum_{i=1}^{L} \sigma_{\zeta_{i k}}^{2} B_{a}^{\zeta i} \Delta X_{a} B_{a}^{\xi i \mathrm{~T}}, \\
\Delta \Lambda_{f}^{(4)}=\sum_{i=1}^{L} \sigma_{\zeta_{i}}^{2} \sum_{k=1}^{q} \bar{\sigma}_{\xi_{k}}^{2} B_{k}^{k i} \Delta X_{a} B_{a}^{k \mathrm{~T}}, \Delta \Lambda_{f}^{(5)}=\sum_{i=1}^{L} \sigma_{\zeta_{i z}}^{2} \sum_{k=1}^{q} \Delta \sigma_{\xi_{k}}^{2} B_{a}^{k i} X_{a} B_{a}^{k i \mathrm{~T}}
\end{gathered}
$$

$$
\begin{gathered}
B_{a}^{\xi k}=\left[\begin{array}{c|c|c}
\Phi_{a}^{\xi k} & (0)_{(n+r+m) \times(n+r+m)} \\
\hdashline(0)_{m \times(n+r+m)} & H_{a}^{\xi k}
\end{array}\right], B_{a}^{\zeta i}=\left[\begin{array}{c|c}
\Phi_{a}^{\zeta i} & (0)_{(n+r+m) \times(n+r+m)} \\
\hdashline(0)_{m \times(n+r+m)} & H_{a}^{\zeta i}
\end{array}\right], \\
B_{a}^{k i}=\left[\begin{array}{cc}
\Phi_{a}^{k i} & (0)_{(n+r+m) \times(n+r+m)} \\
\hdashline(0)_{m \times(n+r+m)} & H_{a}^{k i}
\end{array}\right],
\end{gathered}
$$

then, applying Lemma 2 yields $\Delta \Lambda_{f}^{(1)} \geq 0, \Delta \Lambda_{f}^{(2)} \geq 0, \Delta \Lambda_{f}^{(3)} \geq 0, \Delta \Lambda_{f}^{(4)} \geq 0, \Delta \Lambda_{f}^{(5)} \geq 0$.

Then $\Delta \Lambda_{f}^{(6)}$, and $\Delta \Lambda_{f}^{(7)}$ can be rewritten as

$$
\Delta \Lambda_{f}^{(6)}=D_{a}^{m} \Delta Q_{f} D_{a}^{m \mathrm{~T}}, \Delta \Lambda_{f}^{(7)}=\sum_{i=1}^{L} \sigma_{\zeta_{i}}^{2} D_{a}^{\zeta i} \Delta Q_{f} D_{a}^{\zeta \mathrm{T}}
$$

where

$$
D_{a}^{m}=\left[\begin{array}{c:c}
\Gamma_{a}^{m} & (0)_{(n+r+m) \times(r+m)} \\
\hdashline(0)_{m \times(r+m)} & C_{a}^{m}
\end{array}\right], D_{a}^{\zeta t}=\left[\begin{array}{c:c}
\Gamma_{a}^{\zeta i} & (0)_{(n+r+m) \times(r+m)} \\
\hdashline(0)_{m \times(r+m)} & C_{a}^{\xi_{i}^{i}---}
\end{array}\right]
$$

by using lemma 4 , one can get that

$$
\Delta Q_{f}=\left[\begin{array}{cc}
\Delta Q_{a} & \Delta Q_{a} \\
\Delta Q_{a} & \Delta Q_{a}
\end{array}\right] \geq 0, Q_{f}=\left[\begin{array}{ll}
Q_{a} & Q_{a} \\
Q_{a} & Q_{a}
\end{array}\right] \geq 0,
$$

similar to the proof of $\Delta \Lambda_{f}^{(1)} \geq 0$, we have $\Delta \Lambda_{f}^{(6)} \geq 0$ and $\Delta \Lambda_{f}^{(7)} \geq 0$.

In conclusion, we obtain $\Delta \Lambda_{f}=\Delta \Lambda_{f}^{(1)}+\Delta \Lambda_{f}^{(2)}+\ldots+\Delta \Lambda_{f}^{(7)} \geq 0$, i.e., (60) holds. This completes the proof.

Lemma 7 [26]. Consider the following Lyapunov equation

$$
U=C U C^{\mathrm{T}}+V
$$

where $U, C$ and $V$ are the $n \times n$ matrices, $V$ is a symmetric matrix, $C$ is a stable matrix (i.e., all its eigenvalues are inside the unit circle). If $V \geq 0$, then $U$ is symmetric and unique, and $U \geq 0$.

Theorem 1. For the time-invariant augmented multisensor system (37) and (43), on the basis of 
Hypotheses 1-4, for all admissible uncertainties, the actual CF steady-state Kalman predictors given by (52) are robust, i.e.,

and $P_{a}(-1)$ is the minimal upper bound of $\bar{P}_{a}(-1)$.

$$
\bar{P}_{a}(-1) \leq P_{a}(-1)
$$

Proof. Letting $\Delta P_{a}(-1)=P_{a}(-1)-\bar{P}_{a}(-1)$, from (59) and (60) one has

$$
\Delta P_{a}(-1)=\Psi_{a p} \Delta P_{a}(-1) \Psi_{a p}^{\mathrm{T}}+\Delta^{(c)}, \Delta^{(c)}=\left[I_{n+r+m},-K_{a p}\right] \Delta \Lambda_{f}\left[I_{n+r+m},-K_{a p}\right]^{\mathrm{T}}
$$

Similar to the proof of Theorem 1 in [21], the results of (63) can be hold. The proof is completed.

\subsection{Robust CF steady-state Kalman filter and smoother}

For the worst-case time-invariant CF system (37) and (43) with conservative noise statistics $Q_{f}, R_{f}$, and $S_{f}$, based on the actual CF steady-state Kalman one-step predictor $\hat{x}_{a}(t \mid t-1)$, the actual CF steadystate Kalman filter $(N=0)$ and smoother $(N>0) \hat{x}_{a}(t \mid t+N)$ are given as [27]

$$
\begin{gathered}
\hat{x}_{a}(t \mid t+N)=\hat{x}_{a}(t \mid t-1)+\sum_{k=0}^{N} K_{a p}(k) \varepsilon_{a}(t+k), N \geq 0 \\
K_{a}(k)=P_{a}(-1) \Psi_{a p}^{\mathrm{Tk}} H_{a}^{m \mathrm{~T}} Q_{\varepsilon a}^{-1}, k>0, K_{a f}=K_{a}(0)=P_{a}(-1) H_{a}^{m \mathrm{~T}} Q_{\varepsilon a}^{-1}, k=0
\end{gathered}
$$

The conservative steady-state estimation error variances are given as

$$
P_{a}(N)=P_{a}(-1)-\sum_{k=0}^{N} K_{a}(k) Q_{\varepsilon a} K_{a}^{\mathrm{T}}(k), N \geq 0
$$

We can obtain the steady-state filtering and smoothing errors $\tilde{x}_{a}(t \mid t+N)=x_{a}(t)-\hat{x}_{a}(t \mid t+N)$ as [27]

$$
\tilde{x}_{a}(t \mid t+N)=\Psi_{a N} \tilde{x}_{a}(t \mid t-1)+\sum_{\rho=0}^{N}\left[K_{a \rho}^{N w}, K_{a \rho}^{N \nu}\right] \lambda_{f}(t+\rho)
$$

where $\lambda_{f}(t+\rho)$ is defined by (57), and

$$
\begin{gathered}
\Psi_{a N}=I_{(n+r+m) \times(n+r+m)}-\sum_{k=0}^{N} K_{a}(k) H_{a}^{m} \Psi_{a p}^{k}, \\
K_{a \rho}^{N w}=-\sum_{k=\rho+1}^{N} K_{a}(k) H_{a}^{m} \Psi_{a p}^{k-\rho-1}, N>0, \rho=0, \cdots, N-1, K_{a N}^{N w}=0, N \geq 0, \rho=N, \\
K_{a \rho}^{N v}=\sum_{k=\rho+1}^{N} K_{a}(k) H_{a}^{m} \Psi_{a p}^{k-\rho-1} K_{a p}-K_{a}(\rho), N>0, \rho=0, \cdots, N-1, K_{a N}^{N v}=-K_{a}(N), N \geq 0, \rho=N
\end{gathered}
$$

Applying (68) yields that the actual and conservative steady-state estimation error variances are, respectively, computed by

$$
\begin{aligned}
& \bar{P}_{a}(N)=\Psi_{a N} \bar{P}_{a}(-1) \Psi_{a N}^{\mathrm{T}}+\sum_{\rho=0}^{N}\left[K_{a \rho}^{N w}, K_{a \rho}^{N \nu}\right] \bar{\Lambda}_{f}\left[K_{a \rho}^{N w}, K_{a \rho}^{N \nu}\right]^{\mathrm{T}}, N \geq 0 \\
& \bar{P}_{a}(N)=\Psi_{a N} P_{a}(-1) \Psi_{a N}^{\mathrm{T}}+\sum_{\rho=0}^{N}\left[K_{a \rho}^{N w}, K_{a \rho}^{N \nu}\right] \Lambda_{f}\left[K_{a \rho}^{N w}, K_{a \rho}^{N \nu}\right]^{\mathrm{T}}, N \geq 0
\end{aligned}
$$

Theorem 2. Under the conditions of Assumptions 1-4, the actual CF steady-state Kalman filter and smoother given by (65) are robust, i.e.,

and $P_{a}(N)$ is the minimal upper bound of $\bar{P}_{a}(N)$.

$$
\bar{P}_{a}(N) \leq P_{a}(N), N \geq 0
$$

Proof. Letting $\Delta P_{a}(N)=P_{a}(N)-\bar{P}_{a}(N)$, from (70) and (71) one has

$$
\Delta P_{a}(N)=\Psi_{a N} \Delta P_{a}(-1) \Psi_{a N}^{\mathrm{T}}+\sum_{\rho=0}^{N}\left[K_{a \rho}^{N w}, K_{a \rho}^{N \nu}\right] \Delta \Lambda_{f}\left[K_{a \rho}^{N w}, K_{a \rho}^{N \nu}\right]^{\mathrm{T}}
$$

Applying (61) and (63) yields $\Delta P_{a}(N) \geq 0$, i.e., (72) holds. In a similar way to the proof of Theorem 1, we can prove that $P_{a}(N)$ is the minimal upper bound of $\bar{P}_{a}(N)$, the details are omitted. The proof is completed.

Corollary 1. From the definition $x_{a}(t)=\left[\begin{array}{lll}x^{\mathrm{T}}(t) & w^{\mathrm{T}}(t-1) & y^{(c) \mathrm{T}}(t-1)\end{array}\right]^{\mathrm{T}}$, the robust CF steady-state Kalman estimator of the original system (1)-(4) can be obtained as $\hat{x}(t \mid t+N)=\left[\begin{array}{lll}I_{n} & (0)_{n \times r} & (0)_{n \times m}\end{array}\right] \hat{x}_{a}(t \mid t+N), N=-1, N \geq 0$, and their actual and conservative fusion steady- 
state error variances are, respectively,

$$
\begin{aligned}
& \bar{P}(N)=\left[\begin{array}{lll}
I_{n} & (0)_{n \times r} & (0)_{n \times m}
\end{array}\right] \bar{P}_{a}(N)\left[\begin{array}{lll}
I_{n} & (0)_{n \times r} & (0)_{n \times m}
\end{array}\right]^{\mathrm{T}} \\
& P(N)=\left[\begin{array}{llll}
I_{n} & (0)_{n \times r} & (0)_{n \times m}
\end{array}\right] P_{a}(N)\left[\begin{array}{lll}
I_{n} & (0)_{n \times r} & (0)_{n \times m}
\end{array}\right]^{\mathrm{T}}
\end{aligned}
$$

the robust CF steady-state Kalman estimator $\hat{x}(t \mid t+N)$ are robust, i.e.,

$$
\bar{P}(N) \leq P(N), N=-1, N \geq 0
$$

and $P(N)$ is the minimal upper bound of $\bar{P}(N)$.

\section{Discussion}

This chapter is to verify the effectiveness of the estimators produced by chapter 4 . We chose the uninterruptible power system (UPS) [22] to complete the simulation experiment by MATLAB R2018a. Let the UPS with 1KVA and sample time $10 \mathrm{~ms}$ at half-load operating point.

$$
\begin{aligned}
& x(t+1)=\left(\left[\begin{array}{ccc}
0.9226 & -0.633 & 0 \\
1 & 0 & 0 \\
0 & 1 & 0
\end{array}\right]+\xi_{1}(t)\left[\begin{array}{ccc}
0.1 & 0.3 & 0.02 \\
0.1 & 0.2 & 0.1 \\
0.1 & 0.2 & 0.05
\end{array}\right]+\xi_{2}(t)\left[\begin{array}{ccc}
0.15 & 0.2 & 0.01 \\
0.15 & 0.1 & 0.1 \\
0.15 & 0.1 & 0.04
\end{array}\right]\right) x(t) \\
& +\left(\left[\begin{array}{c}
0.5 \\
0 \\
0.2
\end{array}\right]+\xi_{1}(t)\left[\begin{array}{l}
1 \\
0 \\
0
\end{array}\right]+\xi_{2}(t)\left[\begin{array}{l}
0 \\
0 \\
1
\end{array}\right]\right) w(t) \\
& z_{i}(t)=\left(\left[\begin{array}{lll}
23.737 & 20.287 & 0
\end{array}\right]+\xi_{1}(t)\left[\begin{array}{lll}
1 & 0 & 0
\end{array}\right]+\xi_{2}(t)\left[\begin{array}{lll}
0 & 1 & 0
\end{array}\right]\right) x(t) \\
& +\left(\left[\begin{array}{lll}
0.1 & 0.7 & 0
\end{array}\right]+\xi_{1}(t)\left[\begin{array}{lll}
1 & 0 & 0
\end{array}\right]+\xi_{2}(t)\left[\begin{array}{lll}
0 & 1 & 0
\end{array}\right]\right) w(t)+g_{i}(t), i=1,2,3 \\
& w(t+1)=D w(t)+\eta(t) \\
& y_{i}(t)=\zeta_{i}(t) z_{i}(t)+\left(1-\zeta_{i}(t)\right)\left(1-\zeta_{i}(t-1)\right) z_{i}(t-1), i=1,2,3
\end{aligned}
$$

We take that $L=3, \bar{R}_{\eta}=0.2, R_{\eta}=0.3, \bar{R}_{g_{1}}=0.16, R_{g_{1}}=0.2, \bar{R}_{g_{2}}=0.4, R_{g_{2}}=0.45, \bar{R}_{g_{3}}=0.25, R_{g_{3}}=0.3$, $\bar{\sigma}_{\xi_{1}}^{2}=0.01, \sigma_{\xi_{1}}^{2}=0.02, \bar{\sigma}_{\xi_{2}}^{2}=0.02, \sigma_{\xi_{2}}^{2}=0.03, \varsigma_{1}=0.85, \varsigma_{2}=0.85, \varsigma_{3}=0.9, D=0.905$. In the follow-up phase, the significant simulation results are given.

Table 1 below present the comparison of actual and robust accuracies of local and CF robust steadystate estimator. We see that the accuracy relations meet our expectations.

Table 1. Comparison of actual and robust accuracies

\begin{tabular}{c|c|c|c|c}
\hline & $\begin{array}{c}N=-1 \\
\text { (predictors) }\end{array}$ & $\begin{array}{c}N=0 \\
\text { (filters) }\end{array}$ & $\begin{array}{c}N=1 \\
\text { (one-step smoothers) }\end{array}$ & $\begin{array}{c}N=2 \\
\text { (two-step smoothers) }\end{array}$ \\
\hline $\operatorname{tr} P_{1}(N)$ & 1.3877 & 0.8320 & 0.6355 & 0.5795 \\
\hline $\operatorname{tr} \bar{P}_{1}(N)$ & 0.8388 & 0.5060 & 0.3776 & 0.3385 \\
\hline $\operatorname{tr} P_{2}(N)$ & 1.5254 & 0.9413 & 0.7057 & 0.6307 \\
\hline $\operatorname{tr} \bar{P}_{2}(N)$ & 0.9286 & 0.5751 & 0.4226 & 0.3710 \\
\hline $\operatorname{tr} P_{3}(N)$ & 1.1936 & 0.6779 & 0.5327 & 0.4628 \\
\hline $\operatorname{tr} \bar{P}_{3}(N)$ & 0.7121 & 0.4084 & 0.3119 & 0.3009 \\
\hline $\operatorname{tr} P^{(c)}(N)$ & 0.8507 & 0.4092 & 0.3410 & 0.3352 \\
\hline $\operatorname{tr} \bar{P}^{(c)}(N)$ & 0.3673 & 0.0305 & 0.0317 & 0.0366 \\
\hline
\end{tabular}



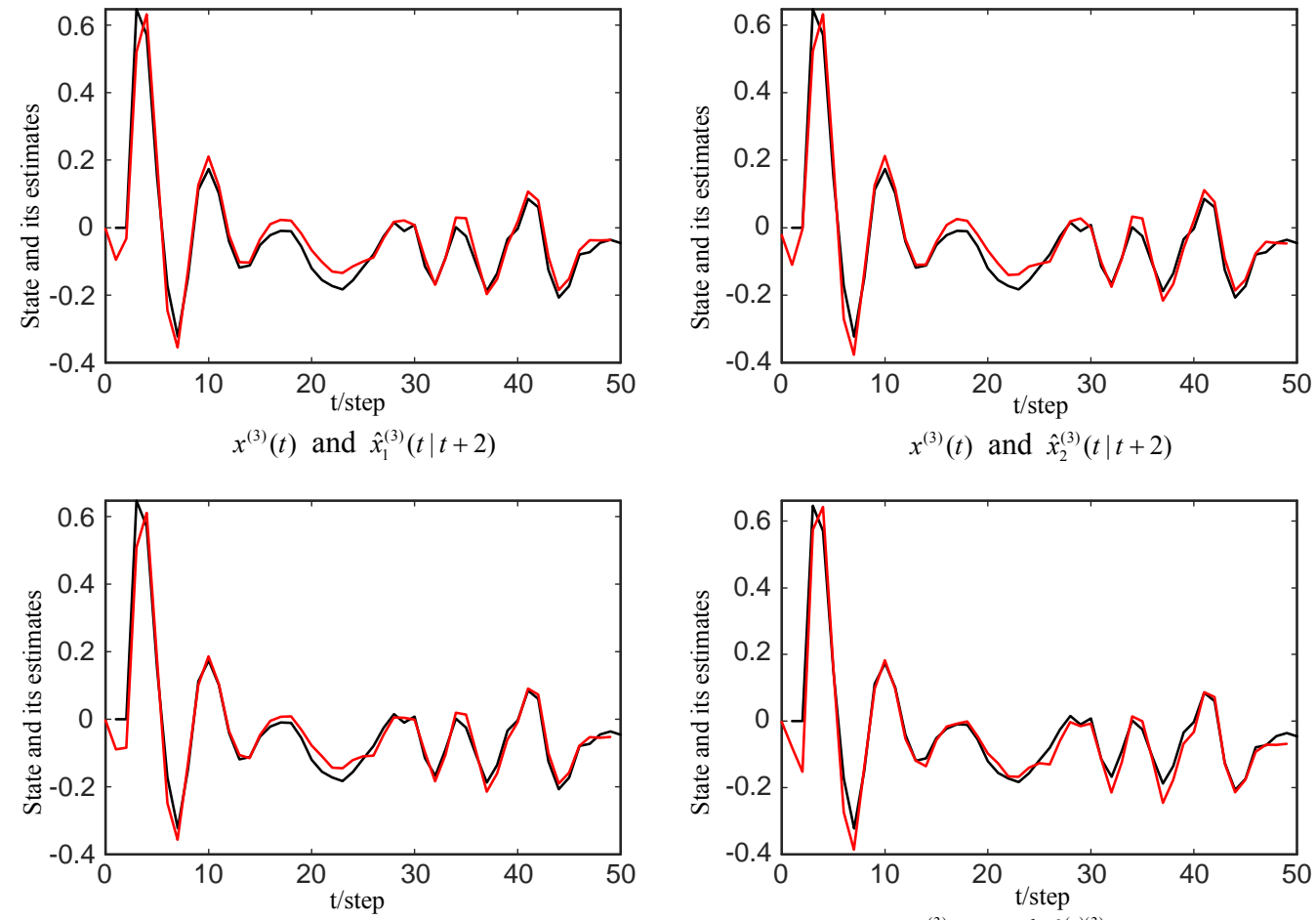

$x^{(3)}(t)$ and $\hat{x}_{3}^{(3)}(t \mid t+2)$

$x^{(3)}(t)$ and $\hat{x}_{2}^{(3)}(t \mid t+2)$

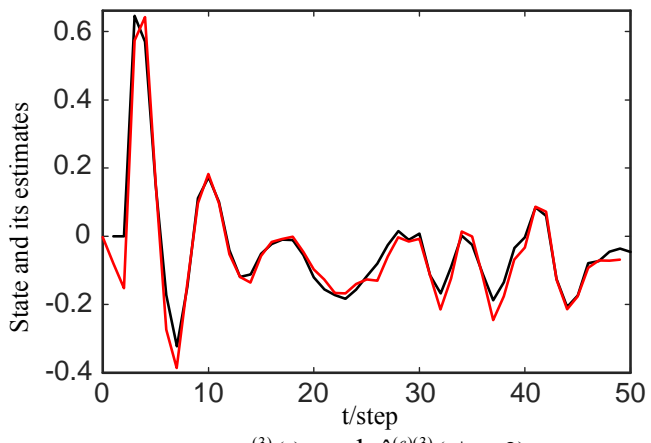

$x^{(3)}(t)$ and $\hat{x}^{(c)(3)}(t \mid t+2)$

the true value of third of actual state $x(t)$

the local and centralized fusion two-step smoother of third component of $x(t)$

Fig. 1 The third component of state $x(t)$ and its actual local and CF two-step smoothers

The results of the tracking experiment are shown in Fig. 1 and Fig. 2. In order to show the best results, we select the third element of state $x(t)$. The tracking results of robust local steady-state two-step smoothers $\hat{x}_{i}^{(3)}(t \mid t+2), i=1,2,3$ and robust CF steady-state two-step smoother $\hat{x}^{(c)(3)}(t \mid t+2)$ are respectively shown in Fig. 1. We see that the CF smoother has better tracking performance compared with the robust local smoothers.

What can be clearly seen in Fig. 2 is that the results of the estimators $\hat{x}^{(c)(3)}(t \mid t+N), N=-1,0,1,2$ gradually get better performance with the increase of $N$. That means that the smoother $\hat{x}^{(c)(3)}(t \mid t+1)$ has better tracking performance than the filter $\hat{x}^{(c)(3)}(t)$ and predictor $\hat{x}^{(c)(3)}(t \mid t-1)$. 

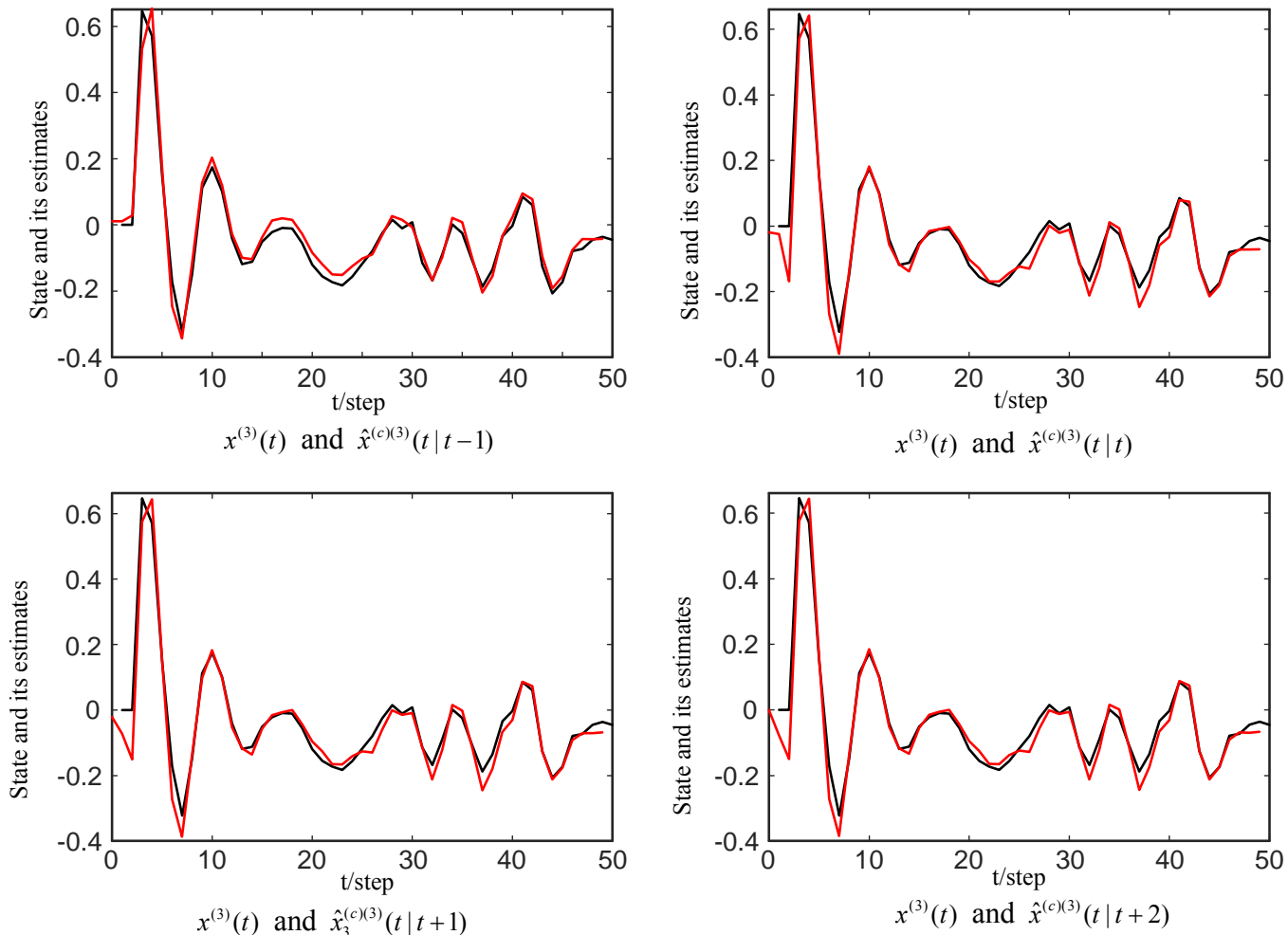

the true value of third component of actual state $x(t)$

the centralized fusion estimator of third component of state $x(t)$

Fig. 2 The third component of state $x(t)$ and its actual centralized fusion estimators

In order to illustrate the robustness of the third component $\hat{x}^{(c)(3)}(t \mid t+1)$ of CF steady-state one-step smoother, we arbitrarily taking three groups of different actual noise variances $\left(\left(\bar{R}_{\eta}\right)^{(h)},\left(\bar{R}_{g_{1}}\right)^{(h)},\left(\bar{R}_{g_{2}}\right)^{(h)},\left(\bar{R}_{g_{3}}\right)^{(h)},\left(\bar{\sigma}_{\xi_{1}}^{2}\right)^{(h)},\left(\bar{\sigma}_{\xi_{2}}^{2}\right)^{(h)}\right), h=1,2,3$ satisfying $(7)$, such that

(1) $\left(\bar{R}_{\eta}\right)^{(1)}=0.102, \quad\left(\bar{R}_{g_{1}}\right)^{(1)}=0.07,\left(\bar{R}_{g_{2}}\right)^{(1)}=0.16,\left(\bar{R}_{g_{3}}\right)^{(1)}=0.11,\left(\bar{\sigma}_{\xi_{1}}^{2}\right)^{(1)}=0.002,\left(\bar{\sigma}_{\xi_{2}}^{2}\right)^{(1)}=0.006$

(2) $\left(\bar{R}_{\eta}\right)^{(1)}=0.131, \quad\left(\bar{R}_{g_{1}}\right)^{(1)}=0.09,\left(\bar{R}_{g_{2}}\right)^{(1)}=0.23,\left(\bar{R}_{g_{3}}\right)^{(1)}=0.16,\left(\bar{\sigma}_{\xi_{1}}^{2}\right)^{(1)}=0.004,\left(\bar{\sigma}_{\xi_{2}}^{2}\right)^{(1)}=0.008$

(3) $\left(\bar{R}_{\eta}\right)^{(1)}=0.162, \quad\left(\bar{R}_{g_{1}}\right)^{(1)}=0.12,\left(\bar{R}_{g_{2}}\right)^{(1)}=0.31,\left(\bar{R}_{g_{3}}\right)^{(1)}=0.25,\left(\bar{\sigma}_{\xi_{1}}^{2}\right)^{(1)}=0.006,\left(\bar{\sigma}_{\xi_{2}}^{2}\right)^{(1)}=0.011$

We easily obtain the corresponding three robust CF steady-state one-step smoothers $\hat{x}_{h}^{(c)}(t \mid t+1), h=1,2,3$, and the corresponding actual and conservative CF steady-state one-step smoothing error variances $\bar{P}_{h}^{(c)}(1)$ and $P^{(c)}(1)$. 


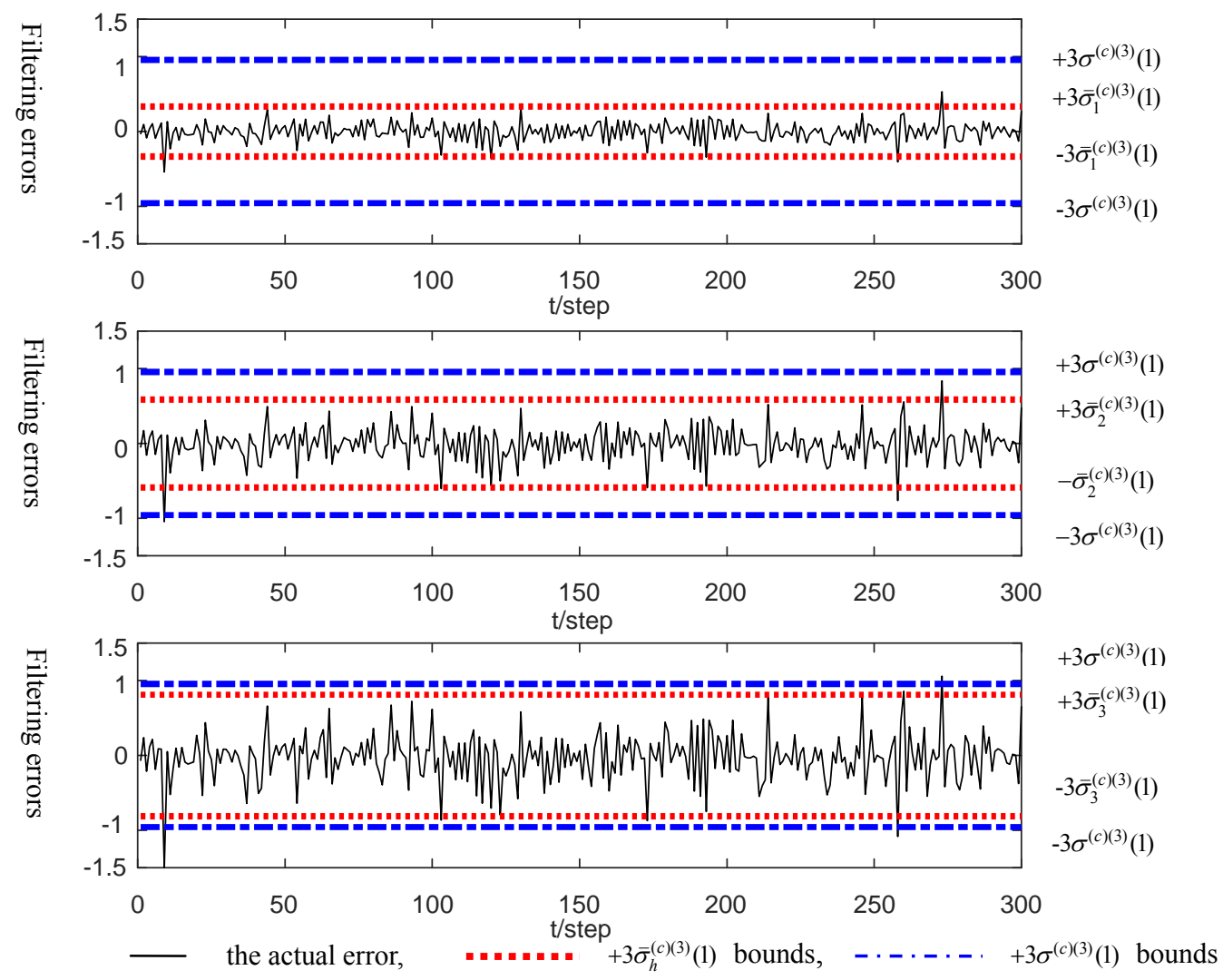

Fig. 3 The actual smoothing error curves of the third component of CF one-step smoother and the corresponding $\pm 3 \bar{\sigma}_{h}^{(c)(3)}(1)$ and $\pm 3 \sigma^{(c)(3)}(1)$ bounds

From Fig. 3 we see that for each fused error curve, over 99 percent of fused one-step smoothing error values lie between $-3 \bar{\sigma}_{h}^{(c)(3)}(1)$ and $+3 \bar{\sigma}_{h}^{(c)(3)}(1)$, and also lie between $-3 \sigma^{(c)(3)}(1)$ and $+3 \sigma^{(c)(3)}(1)$. This verifies the robustness of the robust CF steady-state one-step smoother $\hat{x}^{(c)}(t \mid t+1)$, and the correctness of the actual standard deviations $\bar{\sigma}_{h}^{(c)(3)}(1)$. We also see that when increasing actual noise variances, the actual standard deviations $\bar{\sigma}_{h}^{(c)(3)}(1)$ increase.

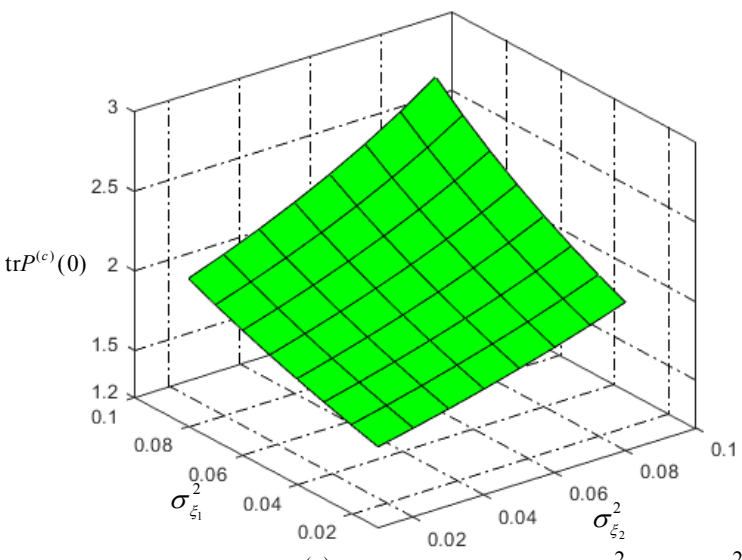

Fig. 4 Changes of $\operatorname{tr} P^{(c)}(0)$ with respect to $\sigma_{\xi_{1}}^{2}$ and $\sigma_{\xi_{2}}^{2}$

To demonstrate the influence of multiplicative noises $\xi_{k}(t), k=1,2$ on the robust accuracy of robust CF steady-state Kalman filter $\hat{x}(t \mid t)$, letting the conservative multiplicative noise variances $\sigma_{\xi_{1}}^{2}$ and $\sigma_{\xi_{2}}^{2}$ 
increase from 0.01 to 0.1 by a step of 0.01 , respectively, the changes of robust accuracy $\operatorname{tr} P^{(c)}(0)$ with respect to $\sigma_{\xi_{1}}^{2}$ and $\sigma_{\xi_{2}}^{2}$ are shown in Fig. 4. We see that when the conservative multiplicative noise variances increase, the values of $\operatorname{tr} P^{(c)}(0)$ increase. This means that when the conservative multiplicative noise variances increase, the robust accuracy of CF steady-state Kalman filter $\hat{x}(t \mid t)$ decrease.

\section{Conclusions}

To address the coloured noise in the system, converted the original system model into a CF system only with uncertain noise variances by using the augmented approach, de-randomization approach and fictitious noise technique. And the process and measurement noises in the CF system are same, which avoids solving the correlation matrix between them. According to the minimax robust estimation principle, on the basis of Hypotheses 1-5, applying the standard Kalman filtering algorithm, the robust CF steadystate Kalman estimators (predictor, filter and smoother) have been proposed. Then their robustness has been proved by using decomposition approach of non-negative definite matrix, and Lyapunov equation approach. A simulation example with application to uninterruptible power system (UPS) with mixed uncertainties has been proposed, which verifies the applicability and correctness of the proposed method.

Abbreviations

\section{Authors' contributions}

\begin{tabular}{|c|l|}
\hline CF & centralized fusion \\
\hline PD & package dropouts \\
\hline UPS & uninterruptible power system \\
\hline
\end{tabular}

Shuang Li-editing, experiments and analysis, Wenqiang Liu-propose the theory model, partial theoretical derivation, Guili Tao-partial theoretical derivation and proof.

\section{Availability of data and materials}

The labeled dataset used to support the findings of this study is available from the corresponding author upon request.

\section{Declarations}

Ethics approval and consent to participate

Not applicable.

\section{Consent for publication}

Not applicable.

\section{Competing interests}

The authors declare that they have no competing interests.

\section{Acknowledgements}

This work is supported by Heilongjiang Provincial Natural Science Foundation of China under Grant No. LH2019F035, by National Natural Science Foundation of China under Grant NSFC-61803148, by Scientific Research Fund of Zhejiang Provincial Education Department under Grant Y202147323, by the Zhejiang Gongshang University General Project of Graduate Research and Innovation Fund in 2021, and is granted from Zhejiang Gongshang University, Zhejiang Provincial Key Laboratory of New Network Standards and Technologies (No. 2013E10012).

\section{References}

1. I. M. Liggins, D. Hall, J. Llinas, Handbook of Multisensor Data Fusion: Theory and Practice, Second Edition. Artech House Radar Libr 39(5), 80-184 (2008)

2. S.L. Sun, Z.L. Deng, Multi-sensor optimal information fusion Kalman filter. Automatica 40, 1017-1203, (2004)

3. B.D.O. Anderson, J. B. Moore, Optimal Filtering. Englewood Cliffs. New Jersey: Prentice Hall (1979)

4. F.L. Lewis, L.H. Xie, P. Dan, Optimal and robust estimation with an introduction to stochastic control theory, Second edition. New York: CRC Press (2008)

5. S.L. Sun, L. Xie, W. Xiao, et al., Optimal linear estimation for systems with multiple packet dropouts. Automatica $\mathbf{4 4}$ (5), 1333-1342 (2008)

6. S. Wang, H. Fang, X. Tian, Robust estimator design for networked uncertain systems with imperfect measurements and uncertain-covariance noises. Neurocomputing 230 (22), 40-47 (2016) 
7. H.L. Tan, B. Shen, H.S. Shu, Robust recursive filtering for stochastic systems with Time-Correlated fading channels, IEEE Trans. Syst. Man Cybern. Syst 99, 1-11 (2021)

8. S.L. Sun, H. Lin, J. Ma, et al., Multi-sensor distributed fusion estimation with applications in networked systems: A review paper. Inf. Fusion 38, 122-134 (2017)

9. N. Nahi, Optimal recursive estimation with uncertain observation. IEEE Trans Inf Theory 15(4), 457-462, (1969)

10. W.J. Qi, P. Zhang, Z.L. Deng, Robust weighted fusion Kalman filters for multisensor time-varying systems with uncertain noise variances. Signal Process 99, 185-200 (2014)

11. J. Ma, S.L. Sun, Optimal linear recursive estimators for stochastic uncertain systems with time-correlated additive noises and packet dropout compensations. Signal Process 176:107704, (2020)

12. J. Hu, G.P. Liu, H. Zhang, et al., On state estimation for nonlinear dynamical networks with random sensor delays and coupling strength under event-based communication mechanism. Information Science 511, 265-283(2020)

13. H. Geng, Z. Wang, Y. Cheng, et al., State estimation under non-Gaussian Lévy and time-correlated additive sensor noises: A modified Tobit Kalman filtering approach. Signal Process 154, 120-128(2019)

14. T. Tian, S.L. Sun, Distributed fusion estimation for multisensor multirate systems with packet dropout compensations and correlated Noises. IEEE Transactions on Systems, Man, and Cybernetics: Systems (99), 1-11(2019)

15. S. Wang, Z. Wang, H. Dong, et al., Recursive state estimation for linear systems with lossy measurements under timecorrelated multiplicative noises. J. Frankl. Inst. 357(3), 1887-1908, (2020)

16. CG. A, HC B, LP. B, Networked fusion estimation with multiple uncertainties and time-correlated channel noise. Inf. Fusion 54, 161-171 (2020)

17. W. Liu, P. Shi, Convergence of Optimal linear estimator with multiplicative and time-correlated additive measurement noises. IEEE Transaction on Automatic Control 64(5), 2190-2197 (2019)

18. R. Caballero-Águila, A. Hermoso-Carazo, J. Linares-Pérez, Centralized filtering and smoothing algorithms from outputs with random parameter matrices transmitted through uncertain communication channels. Digit Signal Process 85, 77-85 (2019)

19. W. Liu, Optimal estimation for discrete-time linear systems in the presence of multiplicative and time-correlated additive measurement noises. IEEE Transactions on Signal Process 63(17), 4583-4593 (2015)

20. W.Q. Liu, X.M. Wang, Z.L. Deng, Robust fusion time-varying Kalman estimators for multisensor networked systems with mixed uncertainties. Int. J. Robust Nonlinear Control. 28 (14), 4139-4174 (2018)

21. W.Q. Liu, G.L. Tao, Y.J. Fan, et al. Robust fusion steady - state filtering for multisensor networked systems with one - step random delay, missing measurements, and uncertain - variance multiplicative and additive white noises. Int. J. Robust and Nonlinear Control 29(14), 1-39 (2019)

22. J. Ma, S.L. Sun, Distributed fusion filter for networked stochastic uncertain systems with transmission delays and packet dropouts. Signal Process 130, 268-278 (2016)

23. N. Li, S. L. Sun, J. Ma. Multi-sensor distributed fusion filtering for networked systems with different delay and loss rates. Digit Signal Process 34, 29-38 (2014)

24. W.A. Zhang, K. Zhou, X.S. Yang, et al., Sequential fusion estimation for networked multisensor nonlinear systems. IEEE Transactions on Industrial Electronics 67(6), 4991-4999 (2020)

25. X. Wang, S.L. Sun, Optimal recursive estimation for networked stochastic uncertain systems with fading measurements and time-correlated channel noises, Journal of Computational and Applied Mathematics 346, 549-565 (2019)

26. T. Kailath, A.H. Sayed, B. Hassibi, Linear estimation, Prentice Hall, New Jersey, Ameriac, (2000)

27. X. J. Sun, G. Yuan, Z. L. Deng, et al. Multi-model information fusion Kalman filtering and white noise deconvolution. Inf. Fusion, 11 (2), 163-173, (2010) 